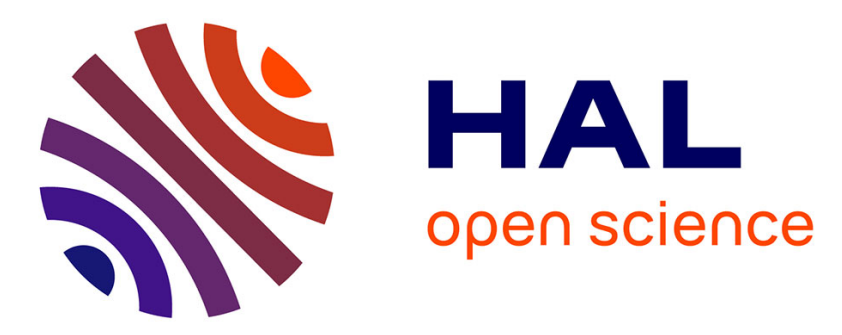

\title{
A pipeline for image based intracardiac CFD modeling and application to the evaluation of the PISA method
}

\author{
Alexandre This, Hernan G Morales, Odile Bonnefous, Miguel Angel \\ Fernández, Jean-Frédéric Gerbeau
}

\section{To cite this version:}

Alexandre This, Hernan G Morales, Odile Bonnefous, Miguel Angel Fernández, Jean-Frédéric Gerbeau. A pipeline for image based intracardiac CFD modeling and application to the evaluation of the PISA method. Computer Methods in Applied Mechanics and Engineering, 2020, 358, pp.112627. 10.1016/j.cma.2019.112627 . hal-02142416

\section{HAL Id: hal-02142416 https://hal.science/hal-02142416}

Submitted on 28 May 2019

HAL is a multi-disciplinary open access archive for the deposit and dissemination of scientific research documents, whether they are published or not. The documents may come from teaching and research institutions in France or abroad, or from public or private research centers.
L'archive ouverte pluridisciplinaire HAL, est destinée au dépôt et à la diffusion de documents scientifiques de niveau recherche, publiés ou non, émanant des établissements d'enseignement et de recherche français ou étrangers, des laboratoires publics ou privés. 


\title{
A pipeline for image based intracardiac CFD modeling and application to the evaluation of the PISA method
}

\author{
Alexandre This ${ }^{\mathrm{a}, \mathrm{b}, \mathrm{c}, *}$, Hernn G. Morales ${ }^{\mathrm{a}}$, Odile Bonnefous ${ }^{\mathrm{a}}$, Miguel A. Fernndez ${ }^{\mathrm{b}, \mathrm{c}}$, Jean-Frdric Gerbeau ${ }^{\mathrm{b}, \mathrm{c}}$ \\ ${ }^{a}$ Medisys, Philips Research, 92150 Suresnes, France \\ ${ }^{b}$ Inria Paris, 75012 Paris, France \\ ${ }^{c}$ Sorbonne Université, UMR 7598 LJLL, 75005 Paris, France
}

\begin{abstract}
Mitral regurgitation is one of the most prevalent valvular heart disease. Proper evaluation of its severity is necessary to choose appropriate treatment. The PISA method, based on Color Doppler echocardiography, is widely used in the clinical setting to estimate various relevant quantities related to the severity of the disease. In this paper, the use of a pipeline to quickly generate image-based numerical simulation of intracardiac hemodynamics is investigated. The pipeline capabilities are evaluated on a database of twelve volunteers. Full pre-processing is achieved completely automatically in 55 minutes, on average, with small registration errors compared to the image spatial resolution. This pipeline is then used to study the intracardiac hemodynamics in the presence of diseased mitral valve. A strong variability among the simulated cases, mainly due to the valve geometry and regurgitation specifics, is found. The results from those numerical simulations is used to assess the potential limitations of the PISA method with respect to different MR types. While the PISA method provides reasonable estimates in the case of a simple circular regurgitation, it is shown that unsatisfying estimates are obtained in the case of non-circular leakage. Moreover, it is shown that the choice of high aliasing velocities can lead to difficulties in quantifying MR.
\end{abstract}

Keywords: Patient-specific, Numerical simulation, Mitral regurgitation

\section{Introduction}

Mitral regurgitation (MR), resulting from pathologies affecting the mitral valve complex [31, is one of the most prevalent valvular heart disease [28, 1]. MR is classified using the Carpentier classification in Type 1 (normal leaflets motion), Type 2 (excessive leaflet motion) and Type 3 (restrictive leaflet motion) 9 . The current clinical guidelines recommend integrating specific, supportive and quantitative features to provide the best MR severity assessment [19, 41, 35]. In that regard, echocardiography plays a significant role in the evaluation of the severity of the MR [35]. Using this imaging technique, effective regurgitant orifice area (EROA), regurgitant volume (RV) and regurgitant fraction (RF) can be estimated using the proximal isovelocity surface area (PISA) method [5]. Although easy to apply, the PISA method suffers from an important number of limitations, due to oversimplifiying assumptions and to the fact that echocardiography is highly physician dependent [32, 41, 19]. We believe that numerical simulation could provide a better insight about the heterogeneous hemodynamics resulting from the different types of MR and help assess the limitations of the current clinical evaluation tools, in particular the PISA technique.

A strong trend in the cardiac modeling community is to personalize models using patient-specific data, geometry and dynamics derived from imaging devices such as computed tomography [12, 25, 23, 17, magnetic resonance imaging 40,38 or echocardiography 6, 38. The reasons behind this trend is multi-fold. First, by exploiting the patient-specific hemodynamics, relevant clinical information can be obtained 14. For example, local informations that cannot be directly obtained on the images, such as the pressures, can be obtained from the simulation results. Moreover, if the predictive power of the models are good enough, medical interventions can be simulated in advance to predict their outcomes and provide tailored care to patients. Finally, by building a big database of in silico cases based on real patients, the medical community

\footnotetext{
${ }^{*}$ Corresponding author
} 
can build a statistical description of the population hemodynamics. Personalizing models, however, brings several additional layers of complexity.

Focusing on intracardiac hemodynamics, several steps are usually required to personalize 3D CFD models based on patient-specific images. The cardiac cavities are first segmented in order to extract the cardiac geometry and dynamics. While this step is nowadays mainly automated, thanks to robust image processing algorithms, the resulting segmented surfaces usually need to go through a serie of operations including mesh cleaning, valve insertion, volumetric mesh generation, surface labeling, inlet and outlet extrusions and refinement of region of interest. Finally, the cardiac dynamics also need to be extracted before being used as boundary condition of the numerical simulations [14. Recent review of patient-specific cardiac flow simulations report that those pre-processing steps are time-consuming, with an order of magnitude of several hours 27, 14.

The objective of this paper is to investigate the use of a pre-processing pipeline [36] designed to speedup the personalization of 3D CFD models. Twelve Real-Time 3D Echocardiography (RT3DE) sequences of healthy volunteers are used to highlight the benefits and limitations of the proposed pipeline. The capabilities of the pipeline to quickly generate realistic CFD simulations are then leveraged to study the intracardiac hemodynamic variability in the presence of diseased mitral valve. Finally, the results from those numerical simulations are used to assess the potential limitations of the PISA method with respect to different MR types.

\section{Image-based CFD modeling}

In this section, the strategy considered to generate image-based CFD simulations is detailed. First, a generic geometry of the left heart and a mathematical model of the intracardiac hemodynamics are presented. The strategy used to automatically adapt this generic model to RT3DE sequences is then described.

\subsection{Generic geometry description}

The computational domain has been created based on the Zygote 3D Human Heart Mode $\sqrt{1}$ a geometrical model based on Computed Tomography and Magnetic Resonance Imaging of a healthy, middle-aged, Caucasian male. From this geometry, the inner layer of cardiac tissue, i.e. the layer in contact with the blood, was extracted. The two right cavities were discarded and the left ventricle and left atrium inner surfaces were used as a basis of the so-called generic geometry. Manual edition of the surface mesh, using the software Materialise 3-Matic V11. $0^{2}$, was performed to add the atrial septum. Surface smoothing was performed to ensure that the surface was free of artifacts. A portion of the ascending aorta was also included in the fluid domain in order to model a short section of blood after the aortic valve location. The resulting fluid domain is shown on Figure 1 a

The mathematical model of the valve, described in Section 2.2 , requires inclusion of the valve geometrical surfaces in open and/or closed configuration. The mitral valve was designed, both in open and closed configuration, using the computer aided design (CAD) software SALOME V7.7.1 ${ }^{3}$ Carpentier's functional classification of MV diseases [9] was used to design three different MV closed-state geometries (Appendix Appendix A to allow the modeling of Type 1, Type 2 and Type 3 MR. On the other hand, the closed aortic valve could be taken directly from the Zygote 3D Human Heart Model and the aortic valve in open-configuration was not included. The valve surfaces were inserted in the full geometrical domain, as illustrated on Figure $1 \mathrm{~b}$,

\subsection{Mathematical modeling of the cardiac hemodynamics}

Consider the domain $\Omega \subset \mathbb{R}^{3}$ in which the fluid is described. The solid layer of tissue surrounding the fluid is denoted by $\partial \Omega_{\mathrm{s}}$. This boundary can be decomposed into the atrium endocardium $\partial \Omega_{\mathrm{s}, \text { at }}$, the ventricular endocardium $\partial \Omega_{\mathrm{s}, \mathrm{v}}$, and the aorta walls $\partial \Omega_{\mathrm{s}, \mathrm{ao}}: \partial \Omega_{\mathrm{s}}=\partial \Omega_{\mathrm{s}, \mathrm{at}} \cup \partial \Omega_{\mathrm{s}, \mathrm{v}} \cup \partial \Omega_{\mathrm{s}, \mathrm{ao}}$. The valve surfaces are denoted by $\Sigma_{\mathrm{ao}, \mathrm{c}}$ (aortic valve in closed configuration), $\Sigma_{\mathrm{mv}, \mathrm{o}}$ (mitral valve in open configuration) and $\Sigma_{\mathrm{mv}, \mathrm{c}}$ (mitral

\footnotetext{
${ }^{1}$ Zygote Media Group, Inc. (UT, USA), https://www.zygote.com/cad-models (last accessed 03 Dec 2018)

${ }^{2}$ https://www.materialise.com/fr/software/3-matic (last accessed 03 Dec 2018)

3 https://www.salome-platform.org/ (last accessed 03 Dec 2018)
} 


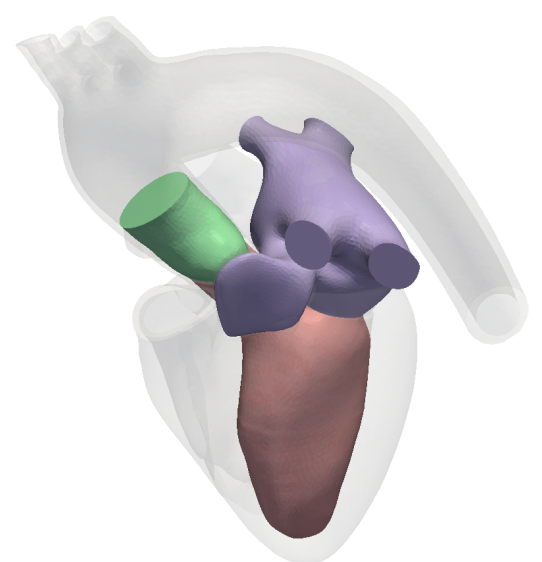

(a)

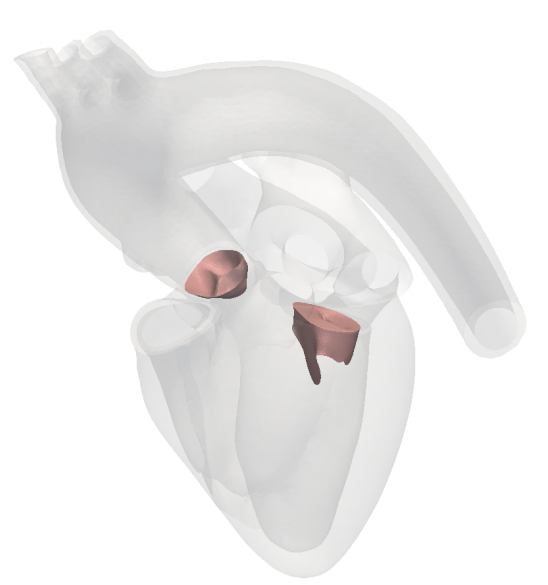

(b)

Figure 1: (a) Fluid domain and (b) immersed valves. On each figure, the Zygote 3D Human Heart Model is displayed in transparency for illustration purpose.

valve in closed configuration). The fluid inlets, cross-section of the four pulmonary veins, are denoted $\partial \Omega_{\mathrm{pv}}$. The fluid outlet, cross-section of the aorta, is denoted $\partial \Omega_{\mathrm{ao}}$.

The blood is modeled as an homogeneous, incompressible, single-phase Newtonian fluid using the NavierStokes equations. The fluid is described by its velocity $\boldsymbol{u}$ and its pressure $p$. The Cauchy stress tensor $\boldsymbol{\sigma}(\boldsymbol{u}, p)=-p \boldsymbol{I}+2 \mu \varepsilon(\boldsymbol{u})$ is defined to characterize the modeled fluid. In this Cauchy stress tensor, $\mu$ is the fluid viscosity, $\boldsymbol{I}$ is the identity tensor and $\varepsilon(\boldsymbol{u})=\frac{1}{2}\left(\boldsymbol{\nabla} \boldsymbol{u}+\boldsymbol{\nabla} \boldsymbol{u}^{T}\right)$ is the symmetric strain rate tensor.

The valves are modeled using the Resistive Immersed Surface (RIS) model originally proposed to model flow through a porous interface [18] and further extended to model the heart valves [4]. The RIS model consists of introducing dissipative surface terms in the N-S momentum equation at the leaflet locations. This model is chosen to allow a 3D representation of the heart valve while keeping the numerical complexity of the model reasonable compared to FSI. We denote by $\delta_{\Sigma_{i}}$ the Dirac measure on the immersed surface $\Sigma_{i}$ and by $R_{\Sigma_{i}}(t)$ the associated scalar resistance. In the limit $R_{\Sigma_{i}} \rightarrow \infty$, this model is equivalent to imposing no-slip boundary condition on the immersed surface. In order to model valve regurgitation, the original scalar resistance term $R_{\Sigma_{i}}(t)$ was redefined as a scalar field $R_{\Sigma_{i}}(\boldsymbol{x}, t)$ to locally control the porous behavior of the valve and allow blood to pass through at specific locations.

The ALE formulation was considered to alleviate the difficulties arising from the large deformation of the cardiac cavities. An arbitrary mapping $\mathcal{A}$ is defined to map the reference domain $\Omega$ into the current spatial domain $\Omega(t)$ at each time instant.

$$
\begin{aligned}
\mathcal{A}: \Omega \times \mathbb{R}^{+} & \rightarrow \Omega(t) \\
(\chi, t) & \rightarrow \boldsymbol{x}
\end{aligned}
$$

Defining $\boldsymbol{w}$ as the fluid domain velocity, the ALE time derivative of a given scalar or tensorial field $f$ is expressed as:

$$
\left.\frac{\partial f}{\partial t}\right|_{\mathcal{A}}=\frac{\partial f}{\partial t}+\boldsymbol{w} \cdot \nabla f
$$

Provided a boundary displacement $\boldsymbol{d}(\boldsymbol{x}, t)$ on $\Gamma \subset \partial \Omega_{\mathrm{s}}$, the displacement of the fluid domain is obtained using an arbitrary lifting operator $\mathcal{L}$ and the fluid domain velocity $\boldsymbol{w}$ can then be recovered. One of the main difficulties of the ALE method is to handle very large deformation of the domain. In that regard, the strategy described by Landajuela et al. 22] was used: the lifted displacements are computed by solving a linear elasticity problem incrementally to enhance the robustness with respect to large deformations. Additionally, the Lam parameters are defined element-wise in such a way that small elements are stiffer than large elements 33 .

The complete mathematical model therefore reads : 
1. Find the domain velocity $\boldsymbol{w}: \Omega \times \mathbb{R}^{+} \rightarrow \mathbb{R}^{3}$ such that, given an arbitrary chosen lifting operator $\mathcal{L}$, a boundary displacement $\boldsymbol{d}(\boldsymbol{x}, t)$ on $\Gamma \subset \partial \Omega_{\mathrm{s}}$ and some possible additional boundary conditions:

$$
\left\{\begin{array}{l}
\boldsymbol{d}=\mathcal{L}\left(\left.\boldsymbol{d}\right|_{\Gamma \subset \partial \Omega_{\mathrm{s}}}\right) \\
\boldsymbol{w}=\frac{\partial \boldsymbol{d}}{\partial t}
\end{array} \quad \text { in } \Omega\right.
$$

2. Find the velocity $\boldsymbol{u}: \Omega \times \mathbb{R}^{+} \rightarrow \mathbb{R}^{3}$ and the pressure $p: \Omega \times \mathbb{R}^{+} \rightarrow \mathbb{R}$ such that, under a certain set of initial and boundary conditions:

$$
\begin{array}{r}
\rho\left(\left.\frac{\partial \boldsymbol{u}}{\partial t}\right|_{\mathcal{A}}+(\boldsymbol{u}-\boldsymbol{w}) \cdot \boldsymbol{\nabla u}\right)-\boldsymbol{\nabla} \cdot \boldsymbol{\sigma}(\boldsymbol{u}, p)+\sum_{i} \delta_{\Sigma_{i}} R_{\Sigma_{i}}(\boldsymbol{x}, t)(\boldsymbol{u}-\boldsymbol{w})=\boldsymbol{f} \\
\text { in } \quad \Omega(t) \\
\boldsymbol{\nabla} \cdot \boldsymbol{u}=0 \quad \text { in } \quad \Omega(t)
\end{array}
$$

\subsection{Time and space discretization}

In what follows, we denote by $\tau$ the time-step length. The time discretization of (3)-(4) is performed via a semi-implicit backward Euler scheme. As regards the discretization in space, a stabilized finite element method is considered. To this purpose, we consider a family of triangulations $\left\{\mathcal{T}_{h}\right\}_{0<h<1}$ of the reference fluid domain $\Omega$ which are fitted to the (reference) valve surfaces $\Sigma_{i}$. The discrete space for the velocity $\boldsymbol{V}_{h} \subset\left[H^{1}(\Omega)\right]^{3}$ is the standard Lagrange finite element space of continuous piecewise affine functions. The pressure space $Q_{h} \subset L^{2}(\Omega)$ is also made of piecewise affine functions which are globally continuous except across $\Sigma_{i}$. The resulting fully discrete method can therefore be written as:

Find $\left(\widehat{\boldsymbol{u}}_{h}^{n}, \widehat{p}_{h}^{n}\right) \in \boldsymbol{V}_{h} \times Q_{h}$ with $\left.\widehat{\boldsymbol{u}}_{h}^{n}\right|_{\partial \Omega_{\mathrm{s}}}=\boldsymbol{w}_{h}^{n}$, such that

$$
\begin{aligned}
\frac{\rho}{\tau}\left[\int_{\Omega^{n}} \boldsymbol{u}_{h}^{n} \cdot \boldsymbol{v}_{h}-\int_{\Omega^{n-1}}\right. & \left.\boldsymbol{u}_{h}^{n-1} \cdot \boldsymbol{v}_{h}\right]+a_{\Omega^{n}, h}^{\mathrm{f}}\left(\boldsymbol{u}_{h}^{n-1}-\boldsymbol{w}_{h}^{n} ;\left(\boldsymbol{u}_{h}^{n}, p_{h}^{n}\right),\left(\boldsymbol{v}_{h}, q_{h}\right)\right) \\
& -\rho \int_{\Omega^{n}}\left(\boldsymbol{\nabla} \cdot \boldsymbol{w}_{h}^{n}\right) \boldsymbol{u}_{h}^{n} \cdot \boldsymbol{v}_{h}+\sum_{i} R_{\Sigma_{i}}^{n} \int_{\Sigma_{i}^{n}}\left(\boldsymbol{u}_{h}^{n}-\boldsymbol{w}_{h}^{n}\right) \cdot \boldsymbol{v}_{h} \\
& =\int_{\Omega^{n}} \boldsymbol{f}^{n} \cdot \boldsymbol{v}_{h}-\int_{\partial \Omega_{\mathrm{pv}}^{n}} P_{\mathrm{pv}}^{n} \boldsymbol{n} \cdot \boldsymbol{v}_{h}-\int_{\partial \Omega_{\mathrm{ao}}^{n}} P_{\mathrm{ao}}^{n} \boldsymbol{n} \cdot \boldsymbol{v}_{h}
\end{aligned}
$$

for all $\left(\widehat{\boldsymbol{v}}_{h}, \widehat{q}_{h}\right) \in \boldsymbol{V}_{h} \times Q_{h}$ with $\left.\widehat{\boldsymbol{v}}_{h}\right|_{\partial \Omega_{\mathrm{s}}}=\mathbf{0}$.

In (5), we have considered the discrete Navier-Stokes tri-linear form

$$
\begin{aligned}
a_{\Omega^{n}, h}^{\mathrm{f}}\left(\boldsymbol{z}_{h} ;\left(\boldsymbol{u}_{h}, p_{h}\right),\left(\boldsymbol{v}_{h}, q_{h}\right)\right)= & \rho \int_{\Omega^{n}} \boldsymbol{z}_{h} \cdot \boldsymbol{\nabla} \boldsymbol{u}_{h} \cdot \boldsymbol{v}_{h}+\frac{\rho}{2} \int_{\Omega^{n}}\left(\boldsymbol{\nabla} \cdot \boldsymbol{z}_{h}\right) \boldsymbol{u}_{h} \cdot \boldsymbol{v}_{h} \\
& +\int_{\Omega^{n}} \boldsymbol{\sigma}\left(\boldsymbol{u}_{h}, p_{h}\right): \nabla \boldsymbol{v}_{h}+\int_{\Omega^{n}} q_{h} \boldsymbol{\nabla} \cdot \boldsymbol{u}_{h} \\
& +\sum_{K \in \mathcal{T}_{h}^{n}} \int_{K} \delta_{h}\left(\rho\left(\boldsymbol{z}_{h} \cdot \boldsymbol{\nabla}\right) \boldsymbol{u}_{h}+\boldsymbol{\nabla} p_{h}\right) \cdot\left(\rho\left(\boldsymbol{z}_{h} \cdot \nabla\right) \boldsymbol{v}_{h}+\boldsymbol{\nabla} q_{h}\right),
\end{aligned}
$$

where the last term stands for the SUPG/PSPG stabilization (see, e.g., 34]).

Remark: In (5), the fluid integrals in the deformed configurations has to be evaluated by composition with the corresponding discrete ALE map. For example, for the the second term we have

$$
\int_{\Omega^{n}} \boldsymbol{u}_{h}^{n-1} \cdot \boldsymbol{v}_{h}=\int_{\Omega^{n}} \widehat{\boldsymbol{u}}_{h}^{n-1} \circ\left(\mathcal{A}_{h}^{n}\right)^{-1} \cdot \widehat{\boldsymbol{v}}_{h} \circ\left(\mathcal{A}_{h}^{n}\right)^{-1} .
$$




\subsection{Initial and boundary conditions}

The fluid is initially considered at rest (6). On the solid surfaces, it is assumed that the fluid has zero velocity relative to the solid boundary $\partial \Omega_{\mathrm{s}}(7)$ (i.e. no-slip BC). At the inlet (pulmonary vein cross-sections), a normal stress is applied to impose a constant static pressure $P_{\mathrm{pv}}$ (8). Finally, a normal stress was also applied at the outlet of the domain (9). As it was shown that proper choice of outlet boundary condition is critical to obtain realistic physiological behavior [39], a Windkessel RCR lumped parameter model was therefore chosen to represent the afterload of the heart related to the arterial system $(10)$. A backflow stabilization based on a local regularization of the fluid velocity is applied at the aortic outlet [16, eventually modifying (9). In (10), the proximal resistance $R_{p}$, distal resistance $R_{d}$, capacitance $C$ and distal pressure $P_{d}(t)$ are parameters of the model that must be chosen appropriately.

$$
\begin{aligned}
& \left.\boldsymbol{u}\right|_{t=0}=\mathbf{0} \quad \text { in } \Omega(0), \\
& \left.\boldsymbol{u}\right|_{\partial \Omega_{\mathrm{s}}}=\boldsymbol{w} \quad \text { on } \partial \Omega_{\mathrm{s}}(t), \\
& \boldsymbol{\sigma}(\boldsymbol{u}, p) \cdot \boldsymbol{n}=-P_{\mathrm{pv}} \boldsymbol{n} \text { on } \partial \Omega_{\mathrm{pv}}(t), \\
& \boldsymbol{\sigma}(\boldsymbol{u}, p) \cdot \boldsymbol{n}=-P_{\mathrm{ao}}(t) \boldsymbol{n}, \quad \text { on } \partial \Omega_{\mathrm{ao}}(t), \\
& P_{\mathrm{ao}}(t)+R_{d} C \frac{d P_{\mathrm{ao}}(t)}{d t}=\left(R_{p}+R_{d}\right) Q(t)+R_{p} R_{d} C \frac{d Q(t)}{d t}+P_{d}+R_{d} C \frac{d P_{d}(t)}{d t},
\end{aligned}
$$

\subsection{Automated patient-specific pipeline}

In this section, a strategy to combine patient-specific data with the hemodynamical model is described. Using the software QLab, 3DQAdvanced Plugin (Philips, Andover, MA), Real-Time 3D echocardiographic images can be processed to obtain sequences of surfaces that depicts the LV surface and its dynamics. Instead of manually processing the segmented surfaces, a methodology is proposed to minimize the amount of preprocessing work. The basic idea is to morph the generic mesh to produce a personalized geometry, an idea borrowed from the field of bone mechanics [13, 8, and previously introduced in [36. The main steps of the pipeline are now presented.

\subsubsection{Generic mesh registration}

A reference frame of the RT3DE sequence is chosen and its associated segmented mesh is considered. The generic mesh, described in Section 2.1. and the segmented mesh exhibit different local geometries. Moreover, their position in space, topology and size are different. As no atrium and aorta exist in the segmented mesh, those two elements will not be used to drive the registration process. Formally, the objective a the registration process is to find a spatial transformation $\Phi$ that maps an object $A$ onto another object $B$. This amounts to minimize the distance between $\Phi(A)$ and $B$ under some appropriately chosen norm.

As the two LV surfaces exhibit positioning differences as well as local surface differences, a two-step strategy is proposed to handle this registration process. First, an initial affine transformation $\Phi_{1}$ will be used to register the main anatomical parts of the generic ventricle to the patient-specific surface. A deformable transformation $\Phi_{2}$ will then be used to deform this coarsely registered generic surface to fit patient-specific surface. The goal of this two-phase strategy is to use as much a priori information as possible in the first phase to simplify the requirements of the deformable registration phase, usually more computationally intensive.

The following assumptions are considered:

- The apex position is known for each LV mesh;

- The aorta outlet position is known for each LV mesh;

- The base to apex distance is known for each LV mesh.

Using those assumptions, the following transformation are computed:

1. Translation of the apex of the generic mesh onto the apex of the segmentation(transformation $\left.\Phi_{1, t}\right)$;

2. Rotation of the generic mesh to align its long axis with the long axis of the segmented ventricle (transformation $\left.\Phi_{1, \text { la }}\right)$ ); 
3. Rotation of the generic mesh to align its aortic out-tract with the segmented ventricle aortic outtract(transformation $\left.\Phi_{1, \text { ao }}\right)$ );

4. Scaling of the generic mesh so that its base to apex distance is equal to the base to apex distance of the segmented ventricle (transformation $\Phi_{1, \mathrm{~s}}$ );

The total affine transformation $\Phi_{1}$ of the generic surface is the composition of all the above transformation:

$$
\Phi_{1}=\Phi_{1, \mathrm{~s}} \circ \Phi_{1, \mathrm{ao}} \circ \Phi_{1, \mathrm{la}} \circ \Phi_{1, t}
$$

As a result of this first registration step, the two ventricle surfaces are considered superimposed with their main anatomical features positioned at approximately the same location. The objective of the second registration step is to obtain a transformation $\Phi_{2}$ which fits the surface of the generic mesh onto the image segmentation. In order to maintain the original generic mesh integrity, several constraints are imposed: the deformable registration should not change the mesh connectivity, and mesh triangle elements should not be degenerated by the transformation. For those reasons, a special interest was given to the group of diffeomorphisms, a special class of maps which enforces those constraints. In this work, the Large Deformation Diffeomorphic Metric Mapping (LDDMM) framework [2, 20, 15] implemented in the open-source software Deformetrica [15] was used.

In this software, $n_{\mathrm{cp}}$ control points $\left(\boldsymbol{q}_{i}\right)_{i=1, \cdots, n_{\mathrm{cp}}}$ are positioned in the ambient $\mathbb{R}^{3}$ space. These control points are associated with a set of vectors $\left(\boldsymbol{\mu}_{i}(t)\right)_{i=1, \cdots, n_{\mathrm{cp}}}$ called momenta where $t \in(0,1)$ is a pseudo-time representing the evolution of the transformation. A dense velocity vector field $\boldsymbol{V}$ is defined in the ambient space by radial basis function $(\mathrm{RBF})$ interpolation, namely,

$$
\boldsymbol{V}_{t}(\boldsymbol{x})=\sum_{i=1}^{n_{\mathrm{cp}}} K\left(\boldsymbol{x}, \boldsymbol{q}_{i}(t)\right) \boldsymbol{\mu}_{i}(t),
$$

where $K(\boldsymbol{x}, \boldsymbol{y})=\exp \left(\frac{\|\boldsymbol{x}-\boldsymbol{y}\|^{2}}{\sigma^{2}}\right)$ is a Gaussian kernel of width $\sigma$. This velocity vector field is integrated to deform the ambient space and the initial control points by solving

$$
\left\{\begin{aligned}
\frac{\partial \boldsymbol{x}}{\partial t} & =\boldsymbol{V}_{t}(\boldsymbol{x}), \\
\boldsymbol{x}(0) & =\boldsymbol{x}_{0} .
\end{aligned}\right.
$$

It was shown that point trajectories are entirely determined by the initial control point positions $\boldsymbol{q}_{i}(0)$ and by the time-varying momentas $\boldsymbol{\mu}_{i}(t)$ [15. It was also shown that such deformation belongs to the class of diffeomorphisms [26]. The registration algorithm aims to find a set of momenta $\boldsymbol{\mu}_{i}(t)$ such that a similarity criterion is minimized. As such set may not be unique, the set which minimizes the deformation kinetic energy is chosen. Using Deformetrica, the transformation $\Phi_{2}$, parameterized by $\left\{\left(\boldsymbol{q}_{i}(0)\right)_{i=1, \cdots, n_{\mathrm{cp}}},\left(\boldsymbol{\mu}_{i}(t)\right)_{i=1, \cdots, n_{\mathrm{cp}}}\right\}$ is obtained.

The total deformation of space is obtained by composition of the affine transformation with the deformable transformation: $\Phi=\Phi_{2} \circ \Phi_{1}$. As both $\Phi_{1}$ and $\Phi_{2}$ are maps defined on the entire Euclidean space $\mathbb{R}^{3}$, the composition $\Phi$ is too. The map $\Phi$ can therefore be applied on the original generic mesh which includes the aorta and the atrium. As a result, the full generic mesh is deformed and its ventricular surface fits the patient-specific ventricular surface. Moreover, this method choice ensures that the resulting map $\Phi$ is smooth and non-degenerate, thus preserving mesh topology, triangle orientation and global quality of the surface.

\subsubsection{Transfer of the dynamics}

Even though a patient-specific geometry can be obtained using the previously computed map $\Phi$, the LV dynamics from the RT3DE sequence cannot be directly applied as simulation boundary conditions for several reasons. First, the time-resolution of the RT3DE sequence depends on several physical factors and is therefore limited. The CFD simulations usually requires a time resolution $(\approx 1000 \mathrm{~Hz})$ that is two orders of magnitude higher than the usual RT3DE time resolution $(\approx 30 \mathrm{~Hz})$. As a result, temporal interpolation of the dynamics is required. Moreover, as the deformable registration is a result of minimizing a global energy functional, the two registered ventricular surfaces are not ensured to be exactly matching. This nonconformity of the two 
surfaces is accentuated by the fact that the resolution of the two surface mesh might also differ. Therefore, a spatial interpolation strategy is also required.

The position of the vertices of the patient-specific ventricular surface is first interpolated in time using cubic splines. The appropriate discrete displacement field is produced by sampling the continuous spline curves using a time discretization fitting the required simulation time-steps. $\boldsymbol{d}_{t_{n} \rightarrow t_{n+1}}^{s}\left(\boldsymbol{x}_{i}\right)$ denotes the displacement vector of the $i$-th vertex of the segmented mesh $s$ at time $t_{n}$ to its position at time $t_{n+1}$. The dynamics of the patient-specific ventricle is then transferred spatially onto the deformed generic mesh vertices using the normalized RBF interpolation framework using a Gaussian RBF $\phi$ parameterized by a smoothing parameter $\sigma:$

$$
\boldsymbol{d}_{t_{n} \rightarrow t_{n+1}}^{g}(\boldsymbol{x})=\frac{\sum_{i} \boldsymbol{d}_{t_{n} \rightarrow t_{n+1}}^{s}\left(\boldsymbol{x}_{i}\right) \phi\left(d_{i}, \sigma\right)}{\sum_{i} \phi\left(d_{i}, \sigma\right)}, \text { with } d_{i}=\left\|\boldsymbol{x}-\boldsymbol{x}_{i}\right\| .
$$

In (13), the superscript $g$ indicates that the displacement field is defined on the generic mesh vertices. Note that the RBF's only need to be computed once for each pair of vertices and can be reused for all time steps. The shape of the Gaussian basis function results in a quick decay of the weighting coefficient allowing to prioritize the closest nodes in the interpolation. The mean edge length of the patient-specific ventricular surface is used as the smoothing parameter $\sigma$ ensuring that each surface vertex is covering a large enough region. Differentiability of the resulting interpolated displacement field is ensured by the choice of Gaussian RBF. The boundary displacement $\boldsymbol{d}_{t_{n} \rightarrow t_{n+1}}^{g}(\boldsymbol{x})$ is used as the boundary condition of (2).

\section{Application to a database of 12 cases and assessment of the PISA method for MR quantifi- cation}

The image-based CFD model presented in the previous section is applied to a database of twelve volunteers. Accuracy and computational effort is shortly investigated. The capabilities of such methodology is then leveraged to study the hemodynamic variability of heart with diseased mitral valve. Finally, results of those numerical simulations are used to assess the potential limitations of the PISA technique.

\subsection{Database description}

Echocardiographic images of twelve healthy volunteers were acquired using an IE33 ultrasound system (Philips, Andover, MA) equipped with a 1-5 MHz transthoracic matrix array transducer (xMATRIX x5-1). RT3DE images were reconstructed as volumes over one cardiac cycle from acquisitions of sub-volumes over four cardiac cycles. An example of such data is depicted on Figure 2a. Using the software QLab, 3DQAdvanced Plugin (Philips, Andover, MA), segmentation and tracking of the left ventricle was automatically performed. As a result, sequences of meshed surfaces depicting the left ventricle surface and dynamics were obtained. Each segmented surfaces are described by a triangular surface mesh that is composed of a constant number of 795 vertices and 1584 triangles. Examples of the resulting mesh in end-diastolic and end-systolic phases are depicted on Figure $2 \mathrm{~b}$.

The sequences averaged 25 frames per cardiac cycle, ranging from 15 to 38. Computing the volume curve of each individual segmented sequence using the algorithm described in [10, the end-systolic volume ranged from $27.92 \mathrm{~mL}$ to $61.96 \mathrm{~mL}$ while the end-diastolic volume ranged from $47.81 \mathrm{~mL}$ to $130.27 \mathrm{~mL}$ across the database.

\subsection{Pipeline analysis on the database cases}

The pipeline was applied on all twelve cases. Regarding the computational effort, all the calculations were done on a computed using a Intel Core i $\%-4810 M Q$ cadenced at $2.8 \mathrm{GHz}, 16 \mathrm{MB}$ of RAM and a NVIDIA Quadro K4100M graphic card. Table 1 reports the computational time required for the pre-processing (i.e. automatic segmentation, registration and dynamics transfer) of the image sequences. To provide the best possible estimation, no other task were launched at the same time on the computer. An average time of 55 minutes was dedicated to the automatic registration and dynamics transfer. The deformable registration was the most time-consuming task as it represented around $77 \%$ of the total pre-processing time. The second most time-consuming task was the spatial interpolation of the data, and more specifically the writing of the data files for all the time steps. Writing 1600 individual velocity files (two cardiac cycles) accounted 


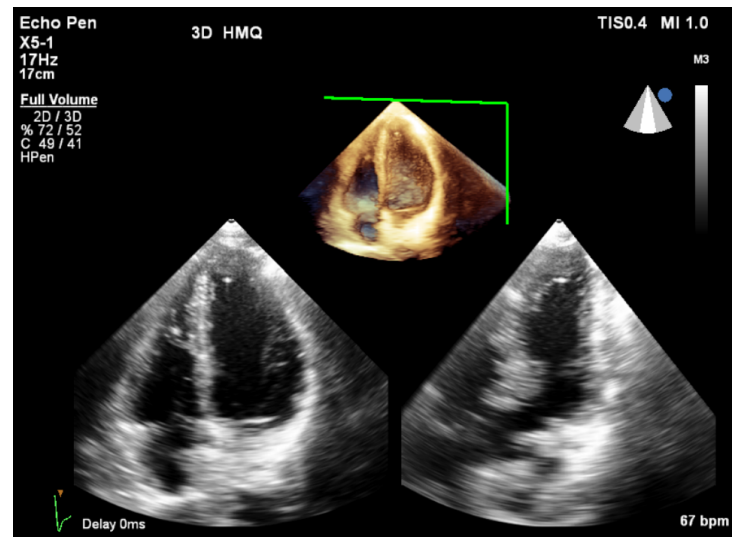

(a)

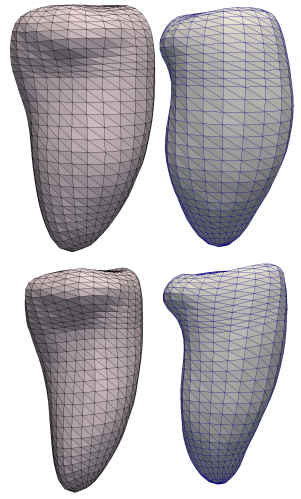

(b)

Figure 2: (a) Transthoracic RT3DE. In the figure, the top part depicts the heart tissue in 3D while the two bottom images depict the tissue information on two orthogonal planes. (b) C12_H end-diastolic (top) and end-systolic (bottom) LV segmentation

for around $22 \%$ of the total time. The affine registration, time interpolation and distance map generation accounted for less than $1 \%$ of the total computational time. After this pre-processing step, the numerical simulations could be performed without any additional manual intervention.

The discrete symmetrical haussdorf distance $d_{\mathrm{m}, \mathrm{s}}\left(S, S^{\prime}\right)$ [3] was computed for all cases and is reported in Table 1 alongside simple statistics, in order to quantify the two-step registration accuracy. The mean symmetric distance between the patient-specific mesh and the registered generic mesh averaged $1.33 \mathrm{~mm}$ $(\sigma=0.08 \mathrm{~mm})$. Direct comparison with the image spatial resolution is difficult as the voxel size of 3D echocardiography are not homogeneous within the image domain. While longitudinal distance between samples is constant, typically $0.5 \mathrm{~mm}$ in the RT3DE sequences used, the azimuth and elevation are described by angular shifts, therefore resulting in lower spatial resolution as the voxel distance to the probe increases. In the available RT3DE sequence, the azimutal voxel size was on the order of $2.15 \mathrm{~mm}$ and the elevation voxel size was on the order of $3.15 \mathrm{~mm}$ in the center of the acquired volumes. The registration distance is therefore small compared to the spatial resolution of the RT3DE images.

\subsection{Synthetic pathological cases generation}

Several synthetic MR cases were produced by combining a RT3DE sequence, a generic mesh including a pathological valve and an arbitrary MR marking strategy. For example, using the RT3DE sequence of patient C01 with a standard flat MV geometry marked by a circular MR region, a Type $1 \mathrm{MR}$ case referred to as C01_T1 was produced. A total of $11 \mathrm{MR}$ cases were designed, including four Type 1, four Type 2 and three Type 3 cases, in order to reproduce MR variability. Four cases were designed for T1 MR as it includes a lot of different geometrical subclasses. Moreover, four cases were designed for T2 MR as it is highly prevalent among the different MR types [1. Specifics about the different synthetic pathological cases can be found in Table 2

\subsection{Numerical simulations of the pathological cases}

Using the deformed generic meshes as the computational domain and the patient-specific LV dynamics as the boundary conditions $\boldsymbol{d}^{n}$ applied on the LV surface, the mathematical model was used to compute the hemodynamics of all the cases with the model parameters available in Appendix Appendix B. Simulations were carried out for one cardiac cycle. The RV and RF was computed for each synthetic pathological cases and can be found in Table 3 A strong variability in the fraction of blood regurgitated was observed as it ranged from approximately $17 \%$ for case C02_T3 to approximately $82 \%$ for case C07_T1. This reflects the variability of the designed synthetic regurgitant cases. The RF was positively correlated with the area of the hole (Figure 3), which is coherent with the current philosophy of assessing MR severity by estimating the EROA as a surrogate value. 


\begin{tabular}{lrr}
\hline Case ID & pre-processing $(\mathrm{s})$ & $d_{\mathrm{m}, \mathrm{s}}\left(S, S^{\prime}\right)(\mathrm{mm})$ \\
\hline C01_H & 3912 & 1.3229 \\
C02_H & 3970 & 1.3775 \\
C03_H & 3271 & 1.3079 \\
C04_H & 2627 & 1.1953 \\
C05_H & 2865 & 1.4080 \\
C06_H & 3631 & 1.1588 \\
C07_H & 3522 & 1.3792 \\
C08_H & 2828 & 1.4564 \\
C09_H & 3457 & 1.2877 \\
C10_H & 3575 & 1.3229 \\
C11_H & 3136 & 1.3325 \\
C12_H & 2812 & 1.3583 \\
\hline Statistics & value $(\mathrm{s})$ & value $(\mathrm{mm})$ \\
\hline$\mu$ & 3314 & 1.3283 \\
$\sigma$ & 432 & 0.0808 \\
min & 2627 & 1.1588 \\
max & 3970 & 1.4564 \\
\hline
\end{tabular}

Table 1: Computation time and symmetric haussdorf distance for all cases and associated statistics

\begin{tabular}{lrr}
\hline Case ID & RV $(\mathrm{mL})$ & RF $(\%)$ \\
\hline C01_T1 & 22.07 & 62.27 \\
C01_T3 & 7.60 & 21.46 \\
C02_T3 & 7.72 & 16.95 \\
C04_T2 & 10.91 & 36.02 \\
C05_T1 & 29.75 & 63.58 \\
C05_T2 & 8.33 & 17.80 \\
C07_T1 & 34.28 & 81.96 \\
C09_T3 & 7.84 & 22.26 \\
C10_T2 & 12.34 & 29.76 \\
C11_T1 & 31.77 & 78.83 \\
C12_T2 & 11.91 & 27.46 \\
\hline
\end{tabular}

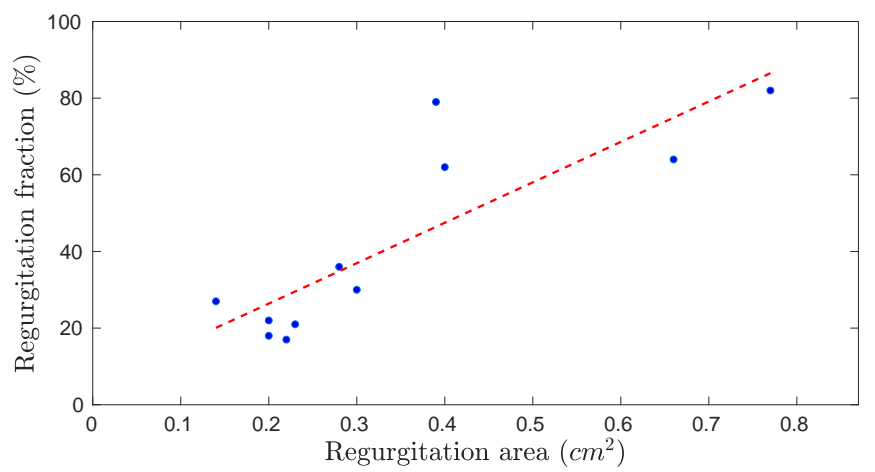

Figure 3: RF plotted as a function of the regurgitation area for the eleven Table 3: RV and RF resulting of the numerical pathological cases. Linear regression (red curve) highlights the positive corsimulations for all pathological cases. relation between the two quantities.

In all the simulated cases, the major features of the diastolic blood flow were similar. As a result of a drop in ventricular pressure, caused by the dilation of the ventricular cavity, a backflow closes the aortic valve. Nearly simultaneously, the MV opens and blood flows from the LA into the LV. Due to the viscosity of the blood and the difference between the mitral inflow velocity and the LV blood velocity, a vortex ring appears at the tip of the MV leaflets. Due to the assymetry of the valve leaflets and their opening angle, the blood entering the LV is directed towards the LV posterior wall. As the vortical structure propagates within the ventricle and reaches the posterior wall, the posterior side of the vortex gets squished whereas the most 


\begin{tabular}{lllll}
\hline Case & MR Type & Comments & Marked area $\left(\mathrm{cm}^{2}\right)$ & Case ID \\
\hline C01 & 1 & Circular hole & 0.40 & C01_T1 \\
& 3 & Anterior leaflet restriction & 0.23 & C01_T3 \\
C02 & 3 & Anterior leaflet restriction & 0.22 & C02_T3 \\
C04 & 2 & P2 Prolapse & 0.28 & C04_T2 \\
C05 & 1 & Two small holes & 0.66 & C05_T1 \\
& 2 & P2 Prolapse & 0.20 & C05_T2 \\
C07 & 1 & Wide slit hole & 0.77 & C07_T1 \\
C09 & 3 & Anterior leaflet restriction & 0.20 & C09_T3 \\
C10 & 2 & P2 Prolapse & 0.30 & C10_T2 \\
C11 & 1 & Wide slit hole & 0.39 & C11_T1 \\
C12 & 2 & P2 Prolapse & 0.14 & C12_T2 \\
\hline
\end{tabular}

Table 2: Synthetic pathological cases description

anterior side fully develops.

Due to the variability of the designed MR, both in term of valve morphology or in term of region marking, the hemodynamics during systole was, however, quite different from case to case. To highlight some of the differences between all those simulations, the blood flow is investigated at mid-systole for all pathological cases and grouped in several categories.

Case C01_T1 was produced by considering the standard MV geometry with a circular hole. As a result, while part of the blood pushed by the LV endocardium is ejected towards the aorta as expected during systole, part of the blood is also directed towards the MV hole and creates a jet of blood with high velocities directed towards the posterior-mid atrium wall (Figure 4a). The isovelocity surfaces of the converging blood are hemispherically shaped, as depicted on Figure $4 \mathrm{~b}$.

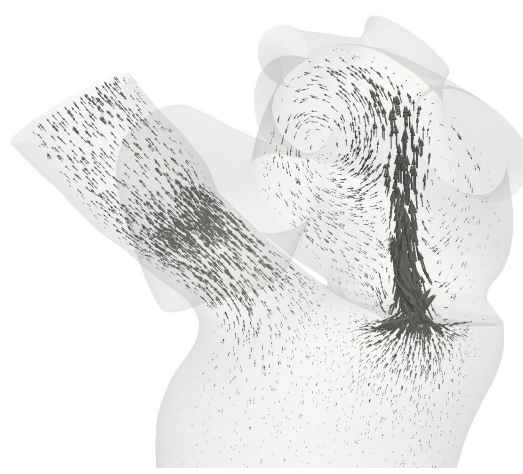

(a)

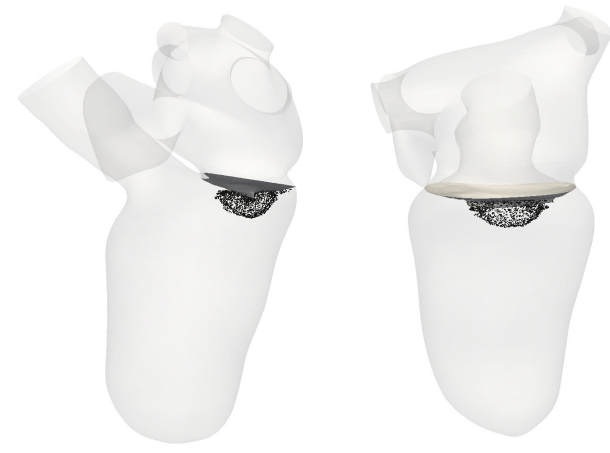

(b)

Figure 4: (a) Blood flow during systole for case C01_T1 and (b) associated isovelocity surface in the convergence region, at an aliasing velocity of $35 \mathrm{~cm} \mathrm{~s}^{-1}$, represented as a point cloud.

Cases C07_T1 and C11_T1 were produced by considering the standard MV geometry with elongated slit holes. As a result of this geometry, the isovelocity surfaces of the converging blood are not hemispherically shaped anymore, they are elongated hemiellipsoids as can be seen on Figure 5 for case C11_T1. 

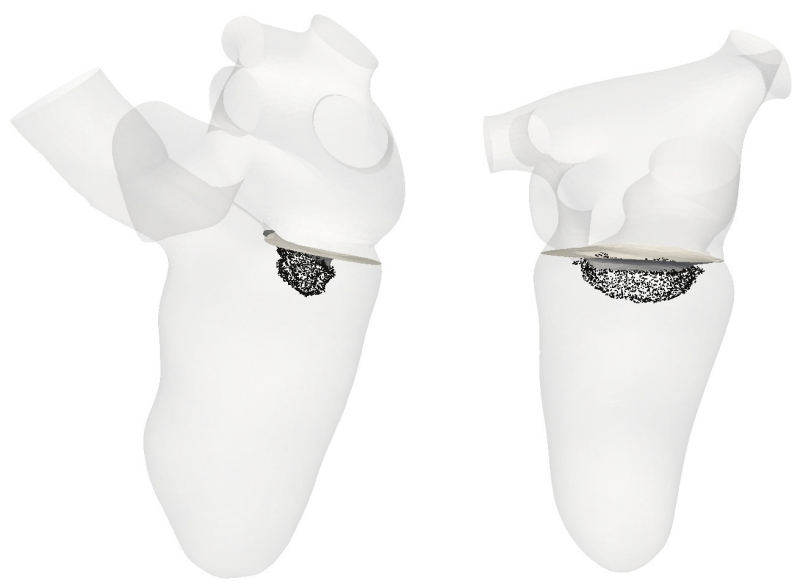

Figure 5: Blood flow during systole for case C11_T1

Case C05_T1 was produced by considering the standard MV geometry and two small holes located at the antero-lateral and postero-medial commissure. Instead of converging towards a single hole, the blood flow splits and part of it passes through one hole while part of it passes through the other. This very specific behavior results in two separate hemispherical isovelocity surfaces (Figure 6).

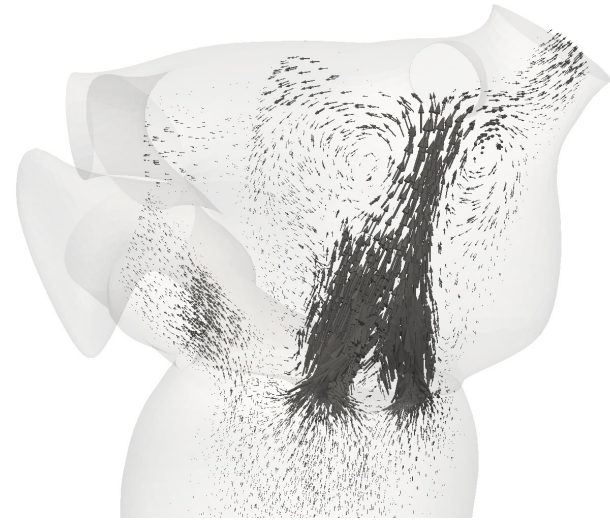

(a)
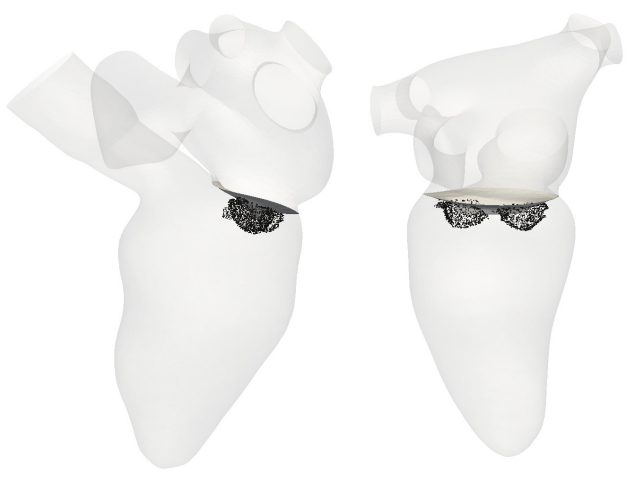

(b)

Figure 6: (a) Blood flow during systole for case C05_T1 and (b) associated isovelocity surface in the convergence region, at an aliasing velocity of $35 \mathrm{~cm} \mathrm{~s}^{-1}$, represented as a point cloud.

Cases C04_T2, C05_T2, C10_T2 and C12_T2 were produced considering the Type 2 MV geometry which included a prolapsed valve with flail posterior leaflets. Contrary to the previously described cases, the resulting MR is highly asymmetric. The blood passes through the MV and the posterior leaflet flail channels the blood flow tangentially with respect to the mitral valve plane. Even though the opening through the MV is rather circular, the isovelocity surfaces are partial hemispheres that are restricted on one side by the presence of the mitral valve (Figure 7). 


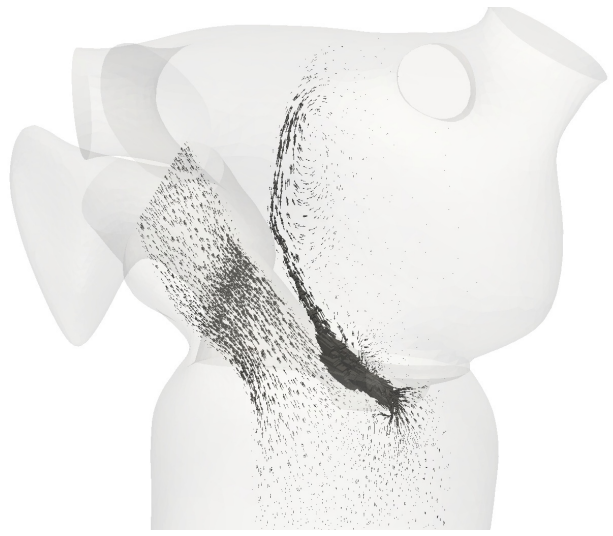

(a)

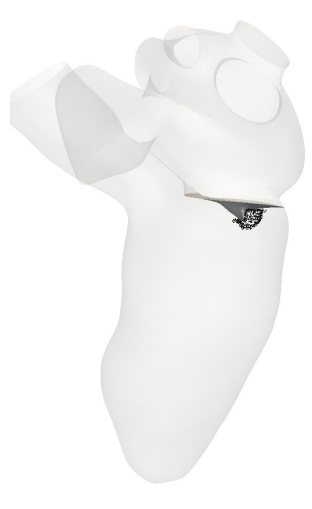

(b)

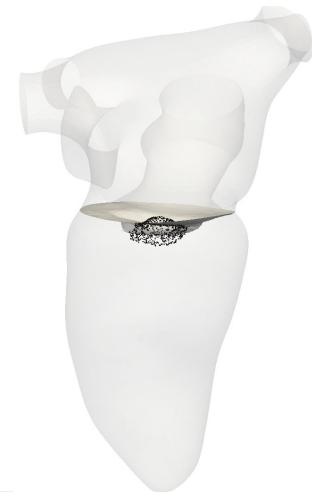

)

Figure 7: (a) Blood flow during systole for case C12_T2 and (b) associated isovelocity surface in the convergence region, at an aliasing velocity of $35 \mathrm{~cm} \mathrm{~s}^{-1}$, represented as a point cloud.

Cases C01_T3, C02_T3 and C09_T3 were produced by considering the Type 3 MV geometry which included a restriction of the anterior leaflet. Similarly to the Type 2 cases described above, the valve configuration is asymmetrical. As the anterior MV leaflet is restricted, a channel orients the flow towards the posterior-inferior atrium wall. As a result of the slit orifice, the isovelocity surfaces are elongated hemiellipsoids, similarly to cases C07_T1 and C11_T1 (Figure 8).

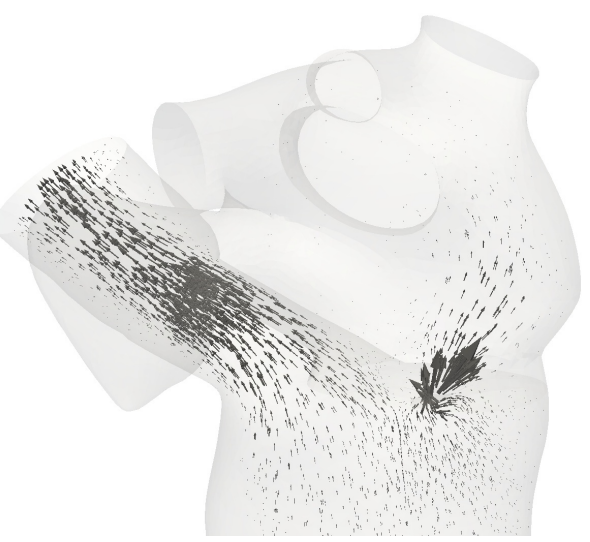

(a)
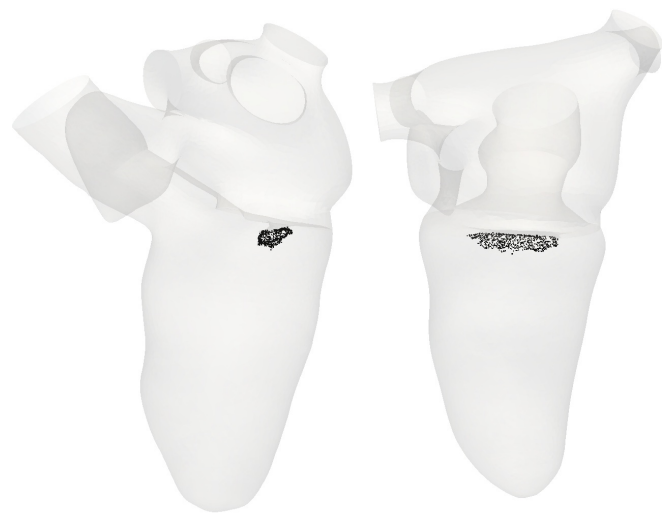

(b)

Figure 8: (a) Blood flow during systole for case C09_T3 and (b) associated isovelocity surface in the convergence region, at an aliasing velocity of $25 \mathrm{~cm} \mathrm{~s}^{-1}$, represented as a point cloud.

The hemodynamics during systole is therefore highly dependent on the underlying MR pathology, MV geometry and orifice. This variability was partly highlighted by the differences of the isovelocity surfaces in the flow convergence region. Indeed, while the circular orifice resulted in an hemispherical isovelocity surface, elongated orifices resulted in hemisellipsoidal isosurfaces, prolapsed valves resulted in partial hemispherical shapes and collection of small regurgitation orifice resulted in a flow convergence region composed of several small isosurfaces. As the PISA method is based on the assumption that the isovelocity surfaces are hemispheric, questions can be raised about potential inaccuracies of the PISA method.

\subsection{Assessment of the PISA method}

It is proposed to assess the accuracy and some of the limitations of the PISA method. We first shortly describe the usual PISA method. Using 2D Color Doppler echocardiography, the physician choses a frame during systole in which the MR is visible and fully developed. He selects a first landmark $\mathcal{L}_{1}$ at the MR center and then chooses an aliasing velocity, denoted $v_{\text {alias }}$, usually ranging from 20 to $60 \mathrm{~cm} \mathrm{~s}^{-1}$ [21, 19, 7, 11]. 
Under the some simplifying assumptions, blood is converging towards the regurgitation and the isovalues of this aliasing velocity are hemispherically distributed around the MR center in the convergence region. The physician chooses a second landmark $\mathcal{L}_{2}$ located on this isovelocity surface and the distance $r_{\text {alias }}$ between the two selected landmarks is computed. The physician computes the area of an hemisphere of radius $r_{\text {alias }}$ and multiplies it with the velocity $v_{\text {alias }}$ to obtain the flow rate at the isovelocity surface level. By dividing this flow rate by the maximal velocity of the regurgitation $v_{\max }$, the physician obtains the EROA. Using the velocity time integral (VTI), the integral of the velocity magnitude at the valve level with respect to time during the MR, the total RV can also be estimated.

To apply the PISA technique, in-silico, the numerical simulations of the pathological cases were processed as follows. The isosurface tool of the Ensight $t^{4}$ software was used to generate 3D isosurfaces at different aliasing velocities ranging from 20 to $60 \mathrm{~cm} \mathrm{~s}^{-1}$ using increments of $10 \mathrm{~cm} \mathrm{~s}^{-1}$. A frame of the sequence was chosen, during systole, in which the isovelocity surfaces were fully developed. The landmark $\mathcal{L}_{1}$, representing the MR center, was defined as the spatial average of five to ten user-defined landmarks to account for some user variability. Since the assumption of hemispherical isovelocities is only valid when the hole is round and the valve is flat, as reported in previous studies [29, 37, there is no unique distance between the MR center and the computed isosurface for the complex MR geometries such as the one presented in this study. Therefore, to imitate the fact that the physician chooses a specific landmark $\mathcal{L}_{2}$ on the isovelocity surface, the methodology described in the following paragraph is adopted. For each aliasing velocity, the distances between the MR center and the triangle centers of the discretized 3D isovelocity surface have been used to produce RV estimates using the PISA method. Those different RV estimates were aggregated into weighted histograms where each estimated value was weighted by the area of its associated triangle to account for non-uniform discretization of the surface. Each sample of this histogram can be thought as one possible choice of landmark $\mathcal{L}_{2}$ by the physician.

The resulting histogram is therefore a probabilistic evaluation of the RV, for which one assumes a uniform probability of picking any landmark $\mathcal{L}_{2}$ on the isosurface. Modes in this histogram represents the fact that a specific RV has a higher probability of being the one estimated by the physician given the shape of the isovelocity surface. The RV estimates of all aliasing velocities can also be aggregated into one single histogram. Both possibilities (either one histogram per aliasing velocity or an aggregated histogram combining all aliasing velocities) was considered. A red vertical line depicting the ground truth RV that needs to be estimated (as reported in Table 3) was added to the figures depicting the histograms to provide an indication on how successful the PISA method would be at assessing MR in each cases. All the histograms can be found in Appendix C, Representative examples are now detailed.

Figure 9 depicts the histogram combining all aliasing velocities for case C01_T1. While the RV estimates are a little bit spread, a strong mode is visible, corresponding roughly to the RV that needs to be estimated. This mode is representative of a nearly hemispherical isovelocity surface. As the case C01_T1 regurgitation results of a circular hole on a nearly flat valve, the PISA method therefore provides a reasonable RV estimate in this case.

\footnotetext{
${ }^{4}$ https://www.ensight.com/ (last accessed 10 Apr 2019)
} 


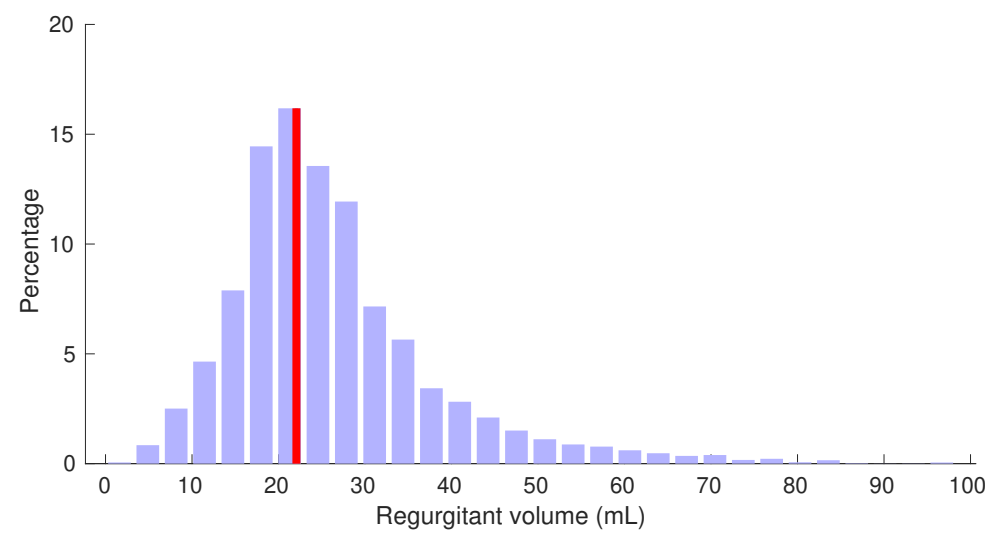

Figure 9: Case C01_T1: Aggregated histogram of all RV estimates at aliasing velocities ranging between 20 to $60 \mathrm{~cm} \mathrm{~s}^{-1}$ by increments of $10 \mathrm{~cm} \mathrm{~s}^{-1}$. The ground-truth RV is overlapping the main mode of the histogram.

Figure 10 depicts the histogram combining all aliasing velocities for case C07_T1. The histogram is dominated by a main mode but one can observe a strong tail of high RV estimates. This is a result of the hemiellipsoid isovelocity surfaces resulting a the slit hole. In this case, the ground truth RV does not overlap the main mode of the histogram revealing the difficulty for the physician to properly quantify MR using the PISA method.

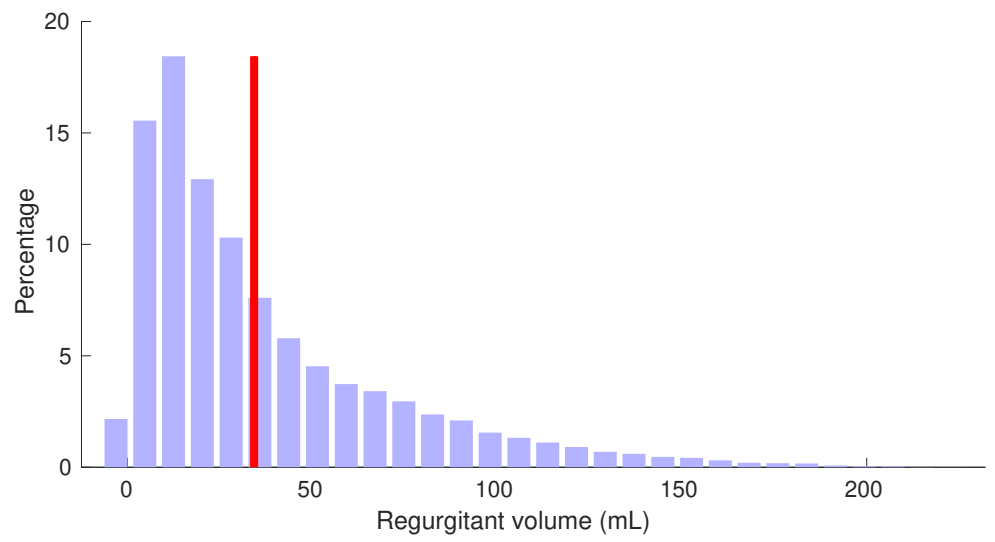

Figure 10: Case 07_T1: Aggregated histogram of all RV estimates at aliasing velocities ranging between 20 to $60 \mathrm{~cm} \mathrm{~s}^{-1}$ by increments of $10 \mathrm{~cm} \mathrm{~s}^{-1}$. The ground-truth RV is greater than the main mode of the histogram.

Figure 11 depicts the histogram combining all aliasing velocities for case C05_T1. Note that C05_T1 is composed of two small regurgitation and, therefore, defining the landmark $\mathcal{L}_{1}$, the MR center, was difficult and had to be chosen among one of the two holes. For this reason, the resulting histogram is difficult to investigate. The histogram is quite uniform in terms of the representativeness of the estimates and one can barely distinguish two modes that might be the result of including the isosurfaces of both regurgitation at the same time. It is difficult to see a relationship between the actual RV and the provided RV estimates. 


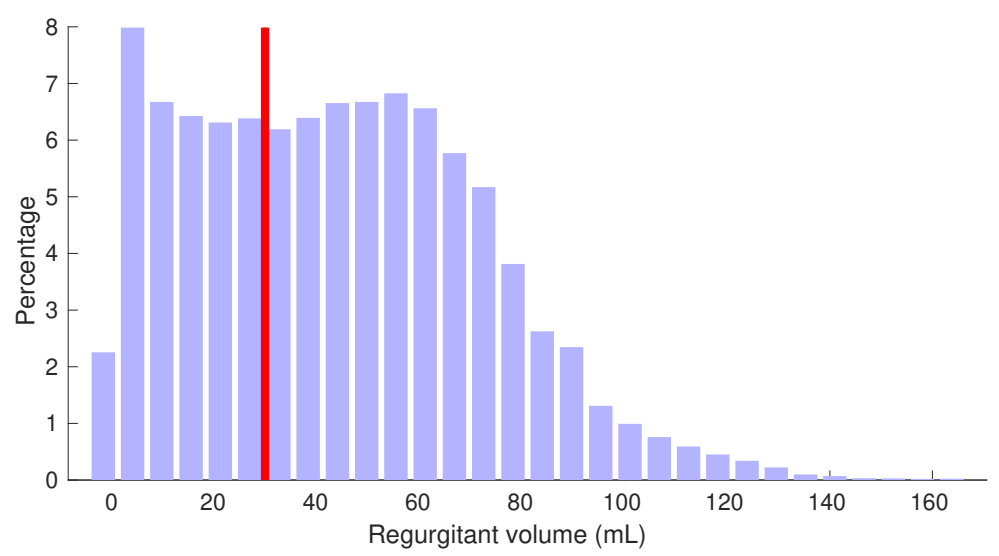

Figure 11: Case C05_T1: Aggregated histogram of all RV estimates at aliasing velocities ranging between 20 to $60 \mathrm{~cm} \mathrm{~s}^{-1}$ by increments of $10 \mathrm{~cm} \mathrm{~s}^{-1}$. Two modes are observed in the histogram The ground-truth RV doesn't math any of those modes.

Figure 12 depict both the histogram combining all aliasing velocities for case C05_T2 and the histogram specific to one aliasing velocity $\left(40 \mathrm{~cm} \mathrm{~s}^{-1}\right)$. A strong mode, relatively representative of the ground truth $\mathrm{RV}$, is visible on Figure 12a. However, one can observe that at specific aliasing velocity, this mode is difficult to observe (Figure 12b). Inspection of the individual histograms associated with specific aliasing velocities Appendix C) reveals that at low aliasing velocities, the RV might be overestimated. At higher aliasing velocities, this overestimation is less and less pronounced. This result indicates that in some cases, it might not be sufficient enough to use a single aliasing velocity to estimate the MR severity.

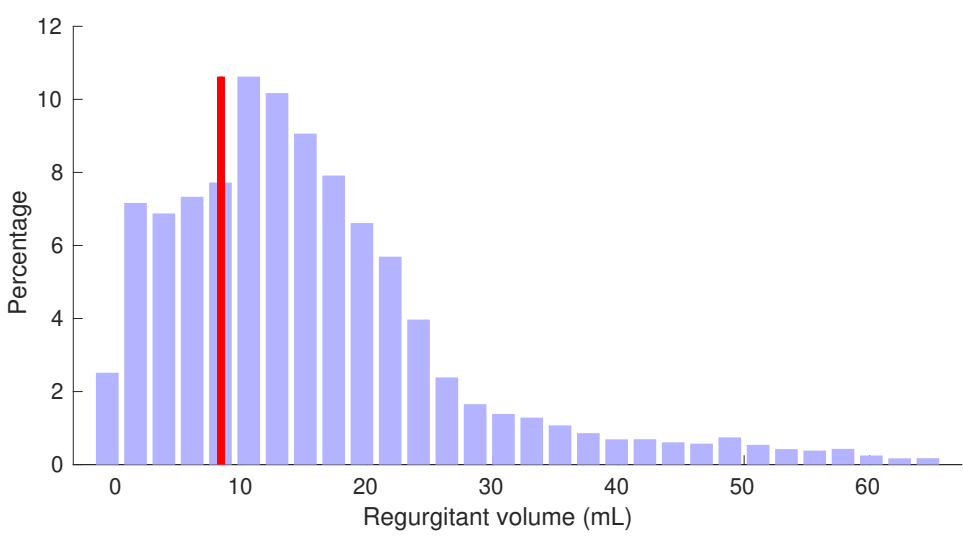

(a)

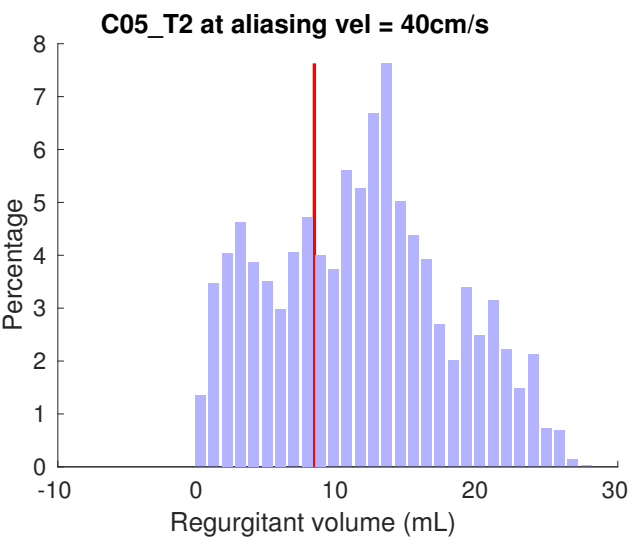

(b)

Figure 12: Case C05_T2: (a) Aggregated histogram of all RV estimates at aliasing velocities ranging between 20 to $60 \mathrm{~cm} \mathrm{~s}^{-1}$ by increments of $10 \mathrm{~cm} \mathrm{~s}^{-1}$ and (b) specific histogram for an aliasing velocity of $40 \mathrm{~cm} \mathrm{~s}^{-1}$

Finally, Figure 13 depicts the histogram combining all aliasing velocities for case C09_T3. Conclusion similar to the Type $1 \mathrm{MR}$ with slit holes can be reached: due to the elongated hole resulting of the anterior leaflet restriction, the histogram contains a main mode with a long tail of high RV estimates. Moreover, the ground truth does not overlap the main mode of the histogram. 


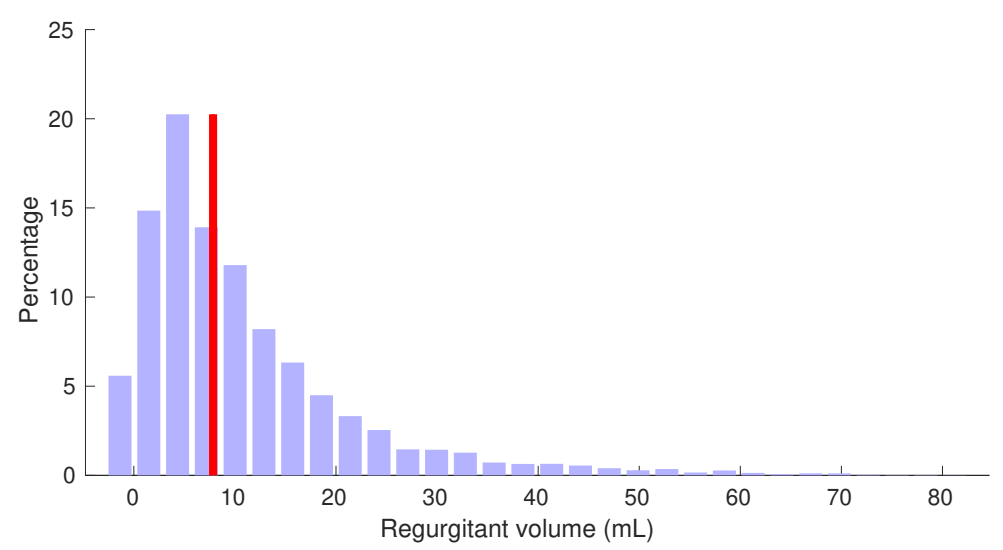

Figure 13: Case C09_T3: Aggregated histogram of all RV estimates at aliasing velocities ranging between 20 to $60 \mathrm{~cm} \mathrm{~s}^{-1}$ by increments of $10 \mathrm{~cm} \mathrm{~s}^{-1}$.

Looking at the spread of the RV estimates across the range of aliasing velocities reveals that lower aliasing velocity leads to a lower spread of the potential RV estimates case-wise. As an example, Figure 14 depicts the histograms of the case C01_T1 placed side by side as a color-coded image. While the RV estimates range from $5 \mathrm{~mL}$ to $50 \mathrm{~mL}$ at an aliasing velocity of $20 \mathrm{~cm} \mathrm{~s}^{-1}$, the RV estimates produced using an aliasing velocity of $60 \mathrm{~cm} \mathrm{~s}^{-1}$ range from $5 \mathrm{~mL}$ to $100 \mathrm{~mL}$. Even though this observation advocates for the use of a small aliasing velocity, one needs to remember that isovelocity surfaces resulting from small aliasing velocities might be disturbed by the aortic outflow. It is therefore important to carefully choose adequate aliasing velocity.

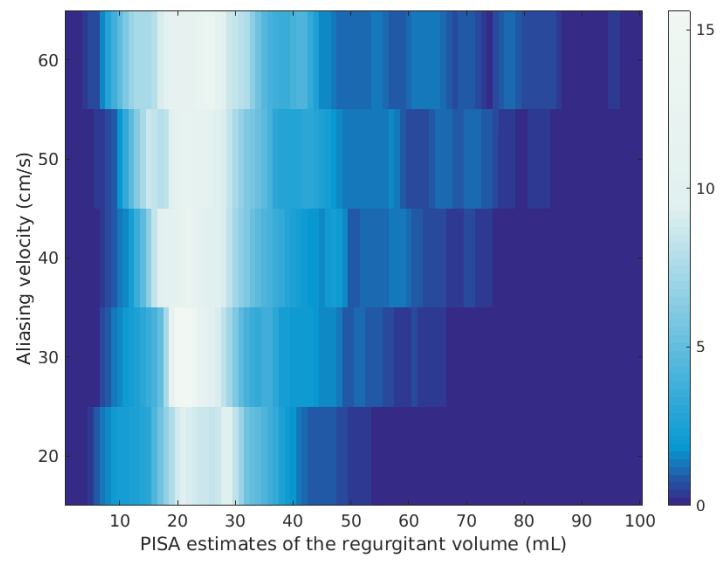

Figure 14: Side by side C01_T1 RV estimates histograms for aliasing velocities ranging from 20 to $60 \mathrm{~cm} \mathrm{~s}^{-1}$. The estimates are more spread in the histograms resulting from high aliasing velocities compared to the histograms resulting from small aliasing velocities.

\section{Conclusion}

In this paper, the use of a pipeline to automatically prepare personalized numerical simulations based on RT3DE image sequences was investigated. This pipeline combines of rigid and deformable registration to morph predefined generic meshes to fit patient-specific LV geometries. Spatio-temporal interpolation of the US data produces appropriate LV surface dynamics on the vertices of the deformed generic meshes.

This pipeline was applied on twelve different RT3DE image sequences. Even though the RT3DE sequences exhibited a strong variability in shape and dynamics of the ventricle, the combination of an affine registration step followed with a deformable registration step allowed to produce patient-specific ventricular surfaces in all cases. Moreover, as specific constraints on the registration were specified, the resulting meshes were all suited for numerical simulations (i.e. no mesh defect were observed in any of the cases). The transfer of 
the LV dynamics was also successful in providing a smooth velocity field of the deformed generic ventricular surface. Regarding the computational effort, the fully automated pre-processing step averaged 55 minutes for the tested database. This is a huge progress compared to the methodology usually reported in the literature. Indeed, we recall here that recent reviews of patient-specific cardiac flow simulations claimed that the pre-processing step usually accounted for more than 20 hours of human effort [27, 14].

Eleven synthetic pathological cases were designed to model various Type 1, Type 2 and Type 3 MR. Investigation of the hemodynamics in those cases revealed heterogeneous systolic blood flow resulting of the underlying pathology and MV geometry. Even though the obtained blood velocity was physiologically coherent, the shortcomings of directly using the RT3DE images as BC for the LV motion is that one strongly relies on the image quality and segmentation artifacts (e.g., non monotonous volume curves during systole or diastole).

Several additional limitations limit the simulation of a fully patient-specific blood flow. First, due to the acquisition process, the atrium is not available in the RT3DE sequences. As a result, the same generic atrium was used for all cases. Moreover, due to image quality, there is no clear definition of internal LV structures such as the trabeculae, papillary muscles or chordae. As a result, smooth ventricular geometry, a strong simplification of reality, was used. Indeed, it has been shown in the past that the papillary muscles [23, trabeculae [23] and chordae 24] have an influence on the intracardiac hemodynamics. Finally, as it is difficult to obtain personalized pressure curves for the pulmonary veins inlet and aortic outlet in a clinical setting, population-derived pressure BC was used.

In this paper, it was shown that the valve shape has strong impact on the blood flow. Therefore, dynamic changes of the valve geometry either during closing of the valve or during systole might introduce additional hemodynamic variability. While the presented work included dynamic motion of the ventricle walls, the MV dynamics was not considered and this might be an oversimplification of the model in some cases. In particular, it is known that the regurgitation and MV dynamics can be highly dynamic in MR caused by mitral prolapse [30. Additional work should be pursued to further characterize the influence of valve dynamics.

Regarding the evaluation of the PISA method, good agreement between the estimated RV using PISA technique and the simulated value was found in the case of a circular hole on a flat mitral valve. However, the PISA method did not offer satisfying estimates for other types of regurgitation. It was also noticed that choosing higher aliasing velocities led to a wider range of potential RV estimates. Those observations highlight the difficulties of properly quantifying MR in a clinical setting. In the presented work, the PISA estimates were obtained using the velocity field of the different numerical simulations. However, in the clinical setting, US Doppler images only provide the component of the velocity field that is aligned with the US beams. Moreover, additional measurements artifacts such as aliasing can perturb the US images. As such, using unaltered velocities coming from simulations might limit the scope of the findings. Nevertheless, this choice allowed to remove the bias of having to choose a specific probe positioning and using unaltered velocities allowed to draw conclusion in a best case scenario.

\section{Acknowledgements}

Funds were supported by a CIFRE Ph.D convention established between INRIA Paris and Philips Research, France.

\section{References}

[1] Andell, P., Li, X., Martinsson, A., Andersson, C., Stagmo, M., Zöller, B., Sundquist, K., Smith, J.G., 2017. Epidemiology of valvular heart disease in a Swedish nationwide hospital-based register study. Heart 103, 1696-1703. URL: http://heart.bmj.com/lookup/doi/10.1136/heartjnl-2017-311472http:// heart.bmj.com/lookup/doi/10.1136/heartjnl-2016-310894, doi:10.1136/heartjnl-2016-310894.

[2] Ashburner, J., 2007. A fast diffeomorphic image registration algorithm. NeuroImage 38, 95113. URL: http://linkinghub.elsevier.com/retrieve/pii/S1053811907005848, doi:10.1016/j. neuroimage.2007.07.007, arXiv:arXiv:1011.1669v3.

[3] Aspert, N., Santa-Cruz, D., Ebrahimi, T., 2002. MESH: measuring errors between surfaces using the Hausdorff distance, in: Proceedings. IEEE International Conference on Multimedia and Expo, 
IEEE. pp. 705-708. URL: http://ieeexplore.ieee.org/document/1035879/, doi:10.1109/ICME. 2002.1035879

[4] Astorino, M., Hamers, J., Shadden, S.C., Gerbeau, J.F.J.F., 2012. A robust and efficient valve model based on resistive immersed surfaces. International Journal for Numerical Methods in Biomedical Engineering 28, 937-959. URL: http://onlinelibrary.wiley.com/doi/10.1002/cnm.1494/fullhttp: //doi.wiley.com/10.1002/cnm.2474, doi:10.1002/cnm.2474.

[5] Baumgartner, H., Falk, V., Bax, J.J., De Bonis, M., Hamm, C., Holm, P.J., Iung, B., Lancellotti, P., Lansac, E., Rodriguez Muñoz, D., Rosenhek, R., Sjögren, J., Tornos Mas, P., Vahanian, A., Walther, T., Wendler, O., Windecker, S., Zamorano, J.L., Roffi, M., Alfieri, O., Agewall, S., Ahlsson, A., Barbato, E., Bueno, H., Collet, J.P., Coman, I.M., Czerny, M., Delgado, V., Fitzsimons, D., Folliguet, T., Gaemperli, O., Habib, G., Harringer, W., Haude, M., Hindricks, G., Katus, H.A., Knuuti, J., Kolh, P., Leclercq, C., McDonagh, T.A., Piepoli, M.F., Pierard, L.A., Ponikowski, P., Rosano, G.M.C., Ruschitzka, F., Shlyakhto, E., Simpson, I.A., Sousa-Uva, M., Stepinska, J., Tarantini, G., Tchétché, D., Aboyans, V., Windecker, S., Aboyans, V., Agewall, S., Barbato, E., Bueno, H., Coca, A., Collet, J.P., Coman, I.M., Dean, V., Delgado, V., Fitzsimons, D., Gaemperli, O., Hindricks, G., Iung, B., Jüni, P., Katus, H.A., Knuuti, J., Lancellotti, P., Leclercq, C., McDonagh, T., Piepoli, M.F., Ponikowski, P., Richter, D.J., Roffi, M., Shlyakhto, E., Simpson, I.A., Zamorano, J.L., Kzhdryan, H.K., Mascherbauer, J., Samadov, F., Shumavets, V., Camp, G.V., Lončar, D., Lovric, D., Georgiou, G.M., Linhartova, K., Ihlemann, N., Abdelhamid, M., Pern, T., Turpeinen, A., Srbinovska-Kostovska, E., Cohen, A., Bakhutashvili, Z., Ince, H., Vavuranakis, M., Temesvári, A., Gudnason, T., Mylotte, D., Kuperstein, R., Indolfi, C., Pya, Y., Bajraktari, G., Kerimkulova, A., Rudzitis, A., Mizariene, V., Lebrun, F., Demarco, D.C., Oukerraj, L., Bouma, B.J., Steigen, T.K., Komar, M., De Moura Branco, L.M., Popescu, B.A., Uspenskiy, V., Foscoli, M., Jovovic, L., Simkova, I., Bunc, M., de Prada, J.A.V., Stagmo, M., Kaufmann, B.A., Mahdhaoui, A., Bozkurt, E., Nesukay, E., Brecker, S.J.D., 2017. 2017 ESC/EACTS Guidelines for the management of valvular heart disease. European Heart Journal 38, 2739-2791. URL: https://academic.oup.com/ eurheartj/article/38/36/2739/4095039, doi:10.1093/eurheartj/ehx391.

[6] Bavo, A.M., Pouch, A.M., Degroote, J., Vierendeels, J., Gorman, J.H., Gorman, R.C., Segers, P., 2016. Patient-specific CFD simulation of intraventricular haemodynamics based on 3D ultrasound imaging. BioMedical Engineering OnLine 15, 107. URL: http://dx.doi.org/10.1186/ s12938-016-0231-9http://biomedical-engineering-online.biomedcentral.com/articles/10. 1186/s12938-016-0231-9, doi:10.1186/s12938-016-0231-9.

[7] Biner, S., Rafique, A., Rafii, F., Tolstrup, K., Noorani, O., Shiota, T., Gurudevan, S., Siegel, R.J., 2010. Reproducibility of Proximal Isovelocity Surface Area, Vena Contracta, and Regurgitant Jet Area for Assessment of Mitral Regurgitation Severity. JACC: Cardiovascular Imaging 3, 235-243. URL: http://dx.doi.org/10.1016/j.jcmg.2009.09.029, doi:10.1016/j.jcmg.2009.09.029

[8] Bucki, M., Lobos, C., Payan, Y., 2010. A fast and robust patient specific Finite Element mesh registration technique: Application to 60 clinical cases. Medical Image Analysis 14, 303-317. doi 10.1016/j.media. 2010.02.003, arXiv:arXiv:1006.2285v1.

[9] Carpentier, A., 1983. Cardiac valve surgery: The 'French correction'. The Journal of thoracic and cardiovascular surgery $86,323-337$.

[10] Cha Zhang, Tsuhan Chen, 2001. Efficient feature extraction for 2D/3D objects in mesh representation, in: Proceedings 2001 International Conference on Image Processing (Cat. No.01CH37205), IEEE. pp. 935-938. URL: http://ieeexplore.ieee.org/document/958278/, doi:10.1109/ICIP.2001.958278.

[11] Chandra, S., Salgo, I.S., Sugeng, L., Weinert, L., Settlemier, S.H., Mor-Avi, V., Lang, R.M., 2011. A three-dimensional insight into the complexity of flow convergence in mitral regurgitation: adjunctive benefit of anatomic regurgitant orifice area. American journal of physiology. Heart and circulatory physiology 301, H1015-H1024. doi:10.1152/ajpheart.00275.2011. 
[12] Chnafa, C., Mendez, S., Nicoud, F., 2014. Image-based large-eddy simulation in a realistic left heart. Comput Fluids 94, 173-187. URL: http://dx.doi.org/10.1016/j.compfluid.2014.01.030, doi 10 . 1016/j.compfluid.2014.01.030.

[13] Couteau, B., Payan, Y., Lavallée, S., 2000. The mesh-matching algorithm: an automatic 3D mesh generator for finite element structures. Journal of Biomechanics 33, 1005-1009. URL: http://linkinghub. elsevier.com/retrieve/pii/S0021929000000555, doi 10.1016/S0021-9290(00)00055-5.

[14] Doost, S.N., Ghista, D., Su, B., Zhong, L., Morsi, Y.S., 2016. Heart blood flow simulation: a perspective review. Biomedical engineering online 15, 101. URL: http://www.ncbi.nlm.nih.gov/pubmed/ 27562639\{\%\}5Cnhttp://www.pubmedcentral.nih.gov/articlerender.fcgi?artid=PMC5000510, doi:10.1186/s12938-016-0224-8.

[15] Durrleman, S., Prastawa, M., Charon, N., Korenberg, J.R., Joshi, S., Gerig, G., Trouvé, A., 2014. Morphometry of anatomical shape complexes with dense deformations and sparse parameters. NeuroImage 101, 35-49. URL: http://linkinghub.elsevier.com/retrieve/pii/S1053811914005205, doi $10.1016 / j$.neuroimage.2014.06.043, arXiv: 15334406

[16] Esmaily Moghadam, M., Bazilevs, Y., Hsia, T.Y., Vignon-Clementel, I.E., Marsden, A.L., 2011. A comparison of outlet boundary treatments for prevention of backflow divergence with relevance to blood flow simulations. Computational Mechanics 48, 277-291. URL: http://link.springer.com/10.1007/ s00466-011-0599-0, doi $10.1007 / \mathrm{s} 00466-011-0599-0$.

[17] Fedele, M., Faggiano, E., Dedè, L., Quarteroni, A., 2017. A patient-specific aortic valve model based on moving resistive immersed implicit surfaces. Biomechanics and Modeling in Mechanobiology 16, 17791803. URL: http://mathicse.epfl.chhttp://link.springer.com/10.1007/s10237-017-0919-1, doi:10.1007/s10237-017-0919-1.

[18] Fernández, M.A., Gerbeau, J.F.J.F., Martin, V., 2008. Numerical simulation of blood flows through a porous interface. ESAIM: Mathematical Modelling and Numerical Analysis 42, 961-990. URL: http: //journals.cambridge.org/abstract\{_\}S0764583X05000166http://www.esaim-m2an.org/10. 1051/m2an:2008031http://journals. cambridge.org/abstract\{\%\}7B\{_\}\{\%\}7DS0764583X05000166, doi:10.1051/m2an:2008031, arXiv:0608007

[19] Grayburn, P.a., 2008. How to measure severity of mitral regurgitation. Postgraduate medical journal 84, 395-402. doi 10.1136/hrt.2005.086462.

[20] Hernandez, M., Bossa, M.N., Olmos, S., 2009. Registration of Anatomical Images Using Paths of Diffeomorphisms Parameterized with Stationary Vector Field Flows. International Journal of Computer Vision 85, 291-306. URL: http://link.springer.com/10.1007/s11263-009-0219-z, doi 10.1007/ s11263-009-0219-z.

[21] Lambert, a.S., 2007. Proximal Isovelocity Surface Area Should Be Routinely Measured in Evaluating Mitral Regurgitation: A Core Review. Anesthesia \& Analgesia 105, 940-943. URL: https://insights . ovid.com/crossref?an=00000539-200710000-00008, doi:10.1213/01.ane.0000278084.35122.4e.

[22] Landajuela, M., Vidrascu, M., Chapelle, D., Fernández, M.A., 2016. Coupling schemes for the FSI forward prediction challenge: Comparative study and validation. International Journal for Numerical Methods in Biomedical Engineering URL: http://doi.wiley.com/10.1002/cnm.2813, doi 10.1002/ cnm.2813.

[23] Lantz, J., Henriksson, L., Persson, A., Karlsson, M., Ebbers, T., 2016. Patient-Specific Simulation of Cardiac Blood Flow From High-Resolution Computed Tomography. Journal of Biomechanical Engineering 138, 121004. URL: http://biomechanical.asmedigitalcollection.asme.org/article.aspx? doi=10.1115/1.4034652, doi $10.1115 / 1.4034652$.

[24] Meschini, V., de Tullio, M.D., Verzicco, R., 2018. Effects of mitral chordae tendineae on the flow in the left heart ventricle. The European Physical Journal E 41, 27. URL: http://link.springer.com/10. 1140/epje/i2018-11634-7, doi:10.1140/epje/i2018-11634-7. 
[25] Mihalef, V., Ionasec, R.I., Sharma, P., Georgescu, B., Voigt, I., Suehling, M., Comaniciu, D., 2011. Patient-specific modelling of whole heart anatomy, dynamics and haemodynamics from four-dimensional cardiac CT images. Interface Focus 1, 286-296. URL: http://rsfs.royalsocietypublishing.org/ cgi/doi/10.1098/rsfs.2010.0036 doi:10.1098/rsfs.2010.0036

[26] Miller, M.I., Trouvé, A., Younes, L., 2006. Geodesic Shooting for Computational Anatomy. Journal of Mathematical Imaging and Vision 24, 209-228. URL: http://link.springer.com/10.1007/ s10851-005-3624-0, doi:10.1007/s10851-005-3624-0, arXiv: NIHMS150003.

[27] Mittal, R., Seo, J.H., Vedula, V., Choi, Y.J., Liu, H., Huang, H.H., Jain, S., Younes, L., Abraham, T., George, R.T., 2016. Computational modeling of cardiac hemodynamics: Current status and future outlook. Journal of Computational Physics 305, 1065-1082. URL: http://dx.doi.org/10.1016/j . jcp.2015.11.022, doi $10.1016 / j \cdot j c p .2015 .11 .022$

[28] Nkomo, V.T., Gardin, J.M., Skelton, T.N., Gottdiener, J.S., Scott, C.G., Enriquez-Sarano, M., 2006. Burden of valvular heart diseases: a population-based study. Lancet 368, 1005-1011. doi 10.1016/ S0140-6736(06)69208-8.

[29] Quaini, A., Canic, S., Guidoboni, G., Glowinski, R., Igo, S.R., Hartley, C.J., Zoghbi, W.a., Little, S.H., 2011. A Three-Dimensional Computational Fluid Dynamics Model of Regurgitant Mitral Valve Flow: Validation Against In Vitro Standards and 3D Color Doppler Methods. Cardiovascular Engineering and Technology 2, 77-89. doi $10.1007 /$ s13239-011-0038-6.

[30] Schwammenthal, E., Chen, C., Benning, F., Block, M., Breithardt, G., Levine, R.A., 1994. Dynamics of mitral regurgitant flow and orifice area. Physiologic application of the proximal flow convergence method: clinical data and experimental testing. Circulation 90, 307-322. URL: http://circ.ahajournals.org/ cgi/doi/10.1161/01.CIR.90.1.307, doi:10.1161/01.CIR.90.1.307.

[31] Silverman, M.E., Hurst, J., 1968. The mitral complex. American Heart Journal 76, 399-418. doi 10. 1016/0002-8703(68)90237-8

[32] Simpson, I.A., Shiota, T., Gharib, M., Sahn, D.J., 1996. Current status of flow convergence for clinical applications: Is it a leaning tower of PISA? Journal of the American College of Cardiology 27, 504-509. URL: http://dx.doi.org/10.1016/0735-1097(95) 00486-6http://linkinghub.elsevier. com/retrieve/pii/0735109795004866 doi:10.1016/0735-1097(95)00486-6.

[33] Stein, K., Tezduyar, T., Benney, R., 2003. Mesh Moving Techniques for Fluid-Structure Interactions With Large Displacements. Journal of Applied Mechanics 70, 58. doi 10.1115/1.1530635.

[34] Tezduyar, T., 1991. Stabilized Finite Element Formulations for Incompressible Flow Computations, volume 28, pp. 1-44. URL: https://linkinghub.elsevier.com/retrieve/pii/S0065215608701534, doi $10.1016 /$ S0065-2156(08)70153-4.

[35] Thavendiranathan, P., Phelan, D., Collier, P., Thomas, J.D., Flamm, S.D., Marwick, T.H., 2012. Quantitative assessment of mitral regurgitation: how best to do it. JACC. Cardiovascular imaging 5, 1161-75. URL:/http://www.sciencedirect.com/science/article/pii/S1936878X12007516http: //www.ncbi.nlm.nih.gov/pubmed/23153917, doi:10.1016/j.jcmg.2012.07.013

[36] This, A., Boilevin-Kayl, L., Morales, H.G., Bonnefous, O., Allain, P., Fernández, M.A., Gerbeau, J.F., 2017. One Mesh to Rule Them All: Registration-Based Personalized Cardiac Flow Simulations, in: International Conference on Functional Imaging and Modeling of the Heart. Springer, Cham, pp. 441-449. URL: http://link.springer.com/10.1007/978-3-319-59448-4\{_\}42, doi 10.1007/ 978-3-319-59448-4_42.

[37] This, A., Morales, H.G., Bonnefous, O., 2016. PROXIMAL ISOVELOCITY SURFACE FOR DIFFERENT MITRAL VALVE HOLE GEOMETRIES, in: Proceedings of the VII European Congress on Computational Methods in Applied Sciences and Engineering (ECCOMAS Congress 2016), Institute of Structural Analysis and Antiseismic Research School of Civil Engineering National Technical University of Athens (NTUA) Greece, Athens. pp. 155-163. URL: http://www.eccomasproceedia.org/ conferences/eccomas-congresses/eccomas-congress-2016/1800, doi:10.7712/100016.1800.6456. 
[38] Vecchi, A.D., Gomez, A., Pushparajah, K., Schaeffter, T., Simpson, J.M., Razavi, R., Penney, G.P., Smith, N.P., Nordsletten, D.A., 2016. A novel methodology for personalized simulations of ventricular hemodynamics from noninvasive imaging data. Computerized Medical Imaging and Graphics 51, 2031. URL: http://dx.doi.org/10.1016/j.compmedimag.2016.03.004, doi $10.1016 / \mathrm{j}$. compmedimag. 2016.03 .004

[39] Vignon-Clementel, I., 2005. A coupled multidomain method for computational modeling of blood flow. Ph.D. thesis. Stanford University.

[40] Votta, E., Le, T.B., Stevanella, M., Fusini, L., Caiani, E.G., Redaelli, A., Sotiropoulos, F., 2013. Toward patient-specific simulations of cardiac valves: State-of-the-art and future directions. Journal of Biomechanics 46, 217-228. URL: http://dx.doi.org/10.1016/j.jbiomech.2012.10.026, doi $10.1016 /$ j.jbiomech.2012.10.026.

[41] ZOGHBI, W., 2003. Recommendations for evaluation of the severity of native valvular regurgitation with two-dimensional and doppler echocardiography. Journal of the American Society of Echocardiography 16, 777-802. URL: http://linkinghub.elsevier.com/retrieve/pii/S0894731703003353, doi:10. 1016/S0894-7317(03)00335-3. 


\section{Appendix A. Pathological closed-state MV geometries}

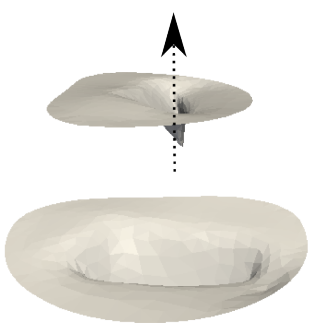

Type 1

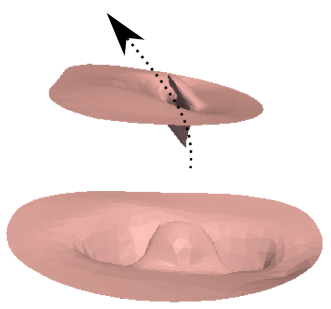

Type 2

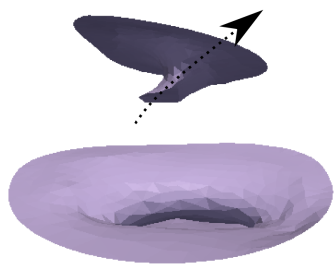

Type 3

Figure A.15: Mitral valve closed-state geometry for Type 1, Type 2 and Type 3 MR. Top and bottom rows of the figures represent different views of the same surface and the black arrows represent potential blood flow resulting the different valve geometries.

\section{Appendix B. Model parameters}

\begin{tabular}{lll}
\hline Type & Parameter & Value \\
\hline Blood parameters & $\rho$ & $1.06 \mathrm{~g} \mathrm{~cm}^{-3}$ \\
& $\mu$ & $0.04 \mathrm{~g} \mathrm{~cm}^{-1} \mathrm{~s}^{-1}$ \\
& $f$ & $\mathbf{0}$ \\
& $P_{\mathrm{pv}}$ & $1.5 \times 10^{4} \mathrm{dyn} \mathrm{cm}^{-2}(\approx 11 \mathrm{mmHg})$ \\
Windkessel parameters & $\left.P_{\text {ao }}\right|_{t=0}$ & $9 \times 10^{4} \mathrm{dyn} \mathrm{cm}^{-2}(\approx 68 \mathrm{mmHg})$ \\
& $R_{p}$ & $150 \mathrm{dyn} \mathrm{s} / \mathrm{cm}^{5}$ \\
& $R_{d}$ & $2300 \mathrm{dyn} \mathrm{s} / \mathrm{cm}^{5}$ \\
& $C$ & $0.9 \mathrm{~cm}^{5} / \mathrm{dyn}^{-2}$ \\
Valve parameters & $P_{d}$ & $0 \mathrm{dyn} \mathrm{cm}^{-2}$ \\
& $R_{\text {max }}$ & $10^{6} \mathrm{~g} / \mathrm{cm}^{2} / \mathrm{s}$ \\
Time discretization & $Q_{\text {backflow }}$ & $2 \mathrm{~cm}^{3} \mathrm{~s}^{-1}$ \\
\hline
\end{tabular}

Table B.4: Model parameters (CGS units)

\section{Appendix C. In-silico PISA histograms}

Appendix C.1. Type 1 

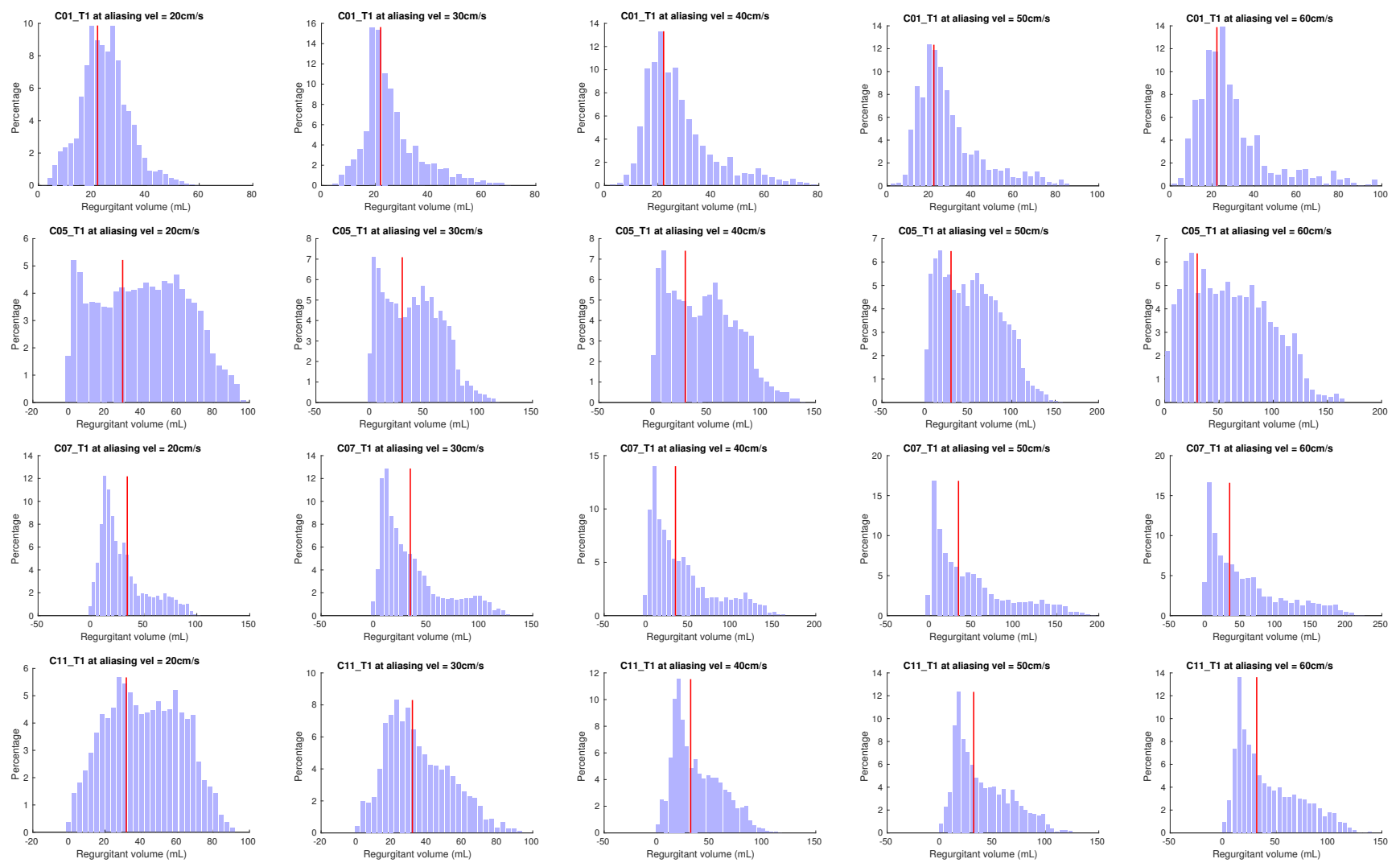

Figure C.16: PISA Estimates histogram for Type 1 cases 
Appendix C.2. Type 2
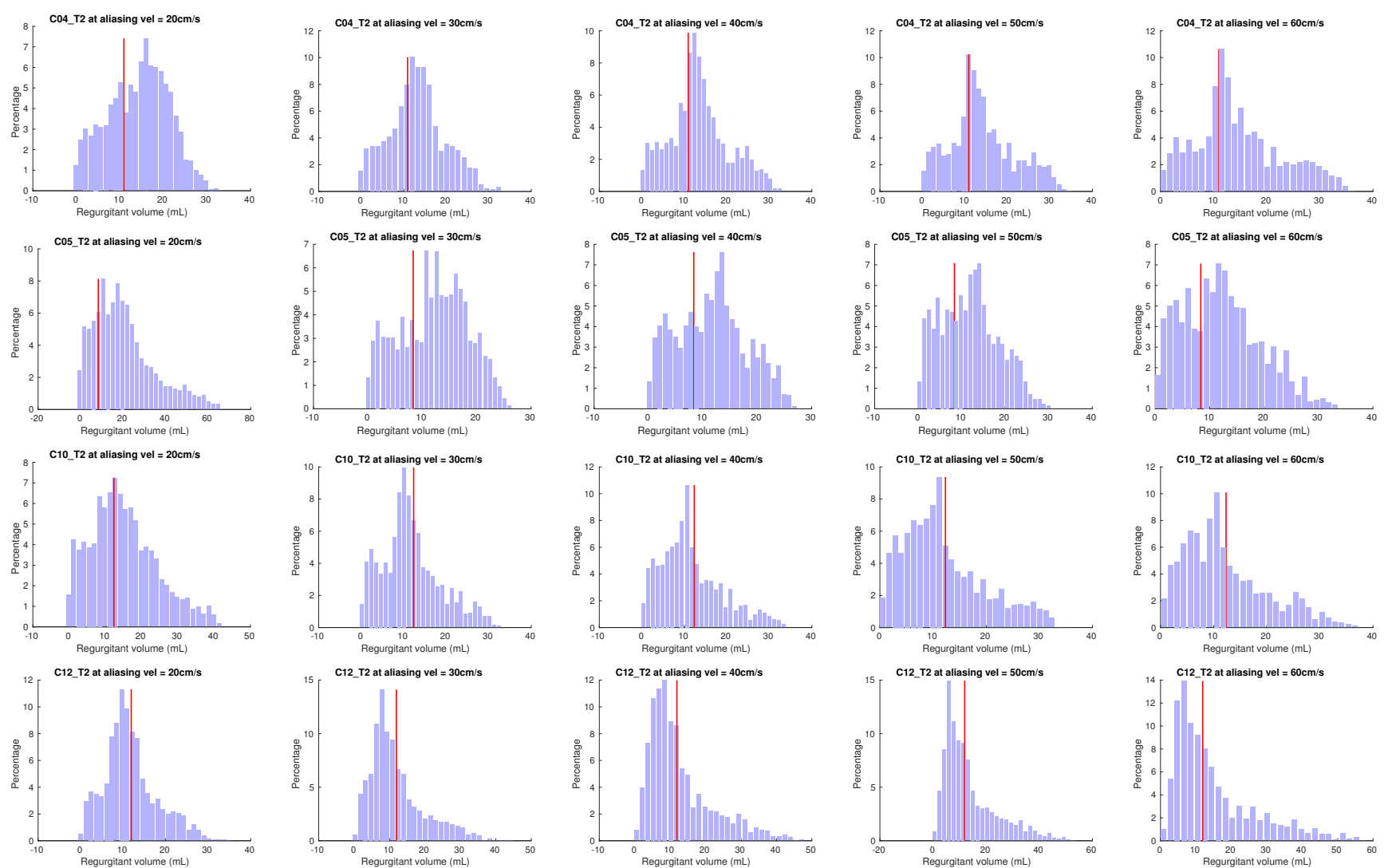

Figure C.17: PISA Estimates histogram for Type 2 cases 
Appendix C.3. Type 3
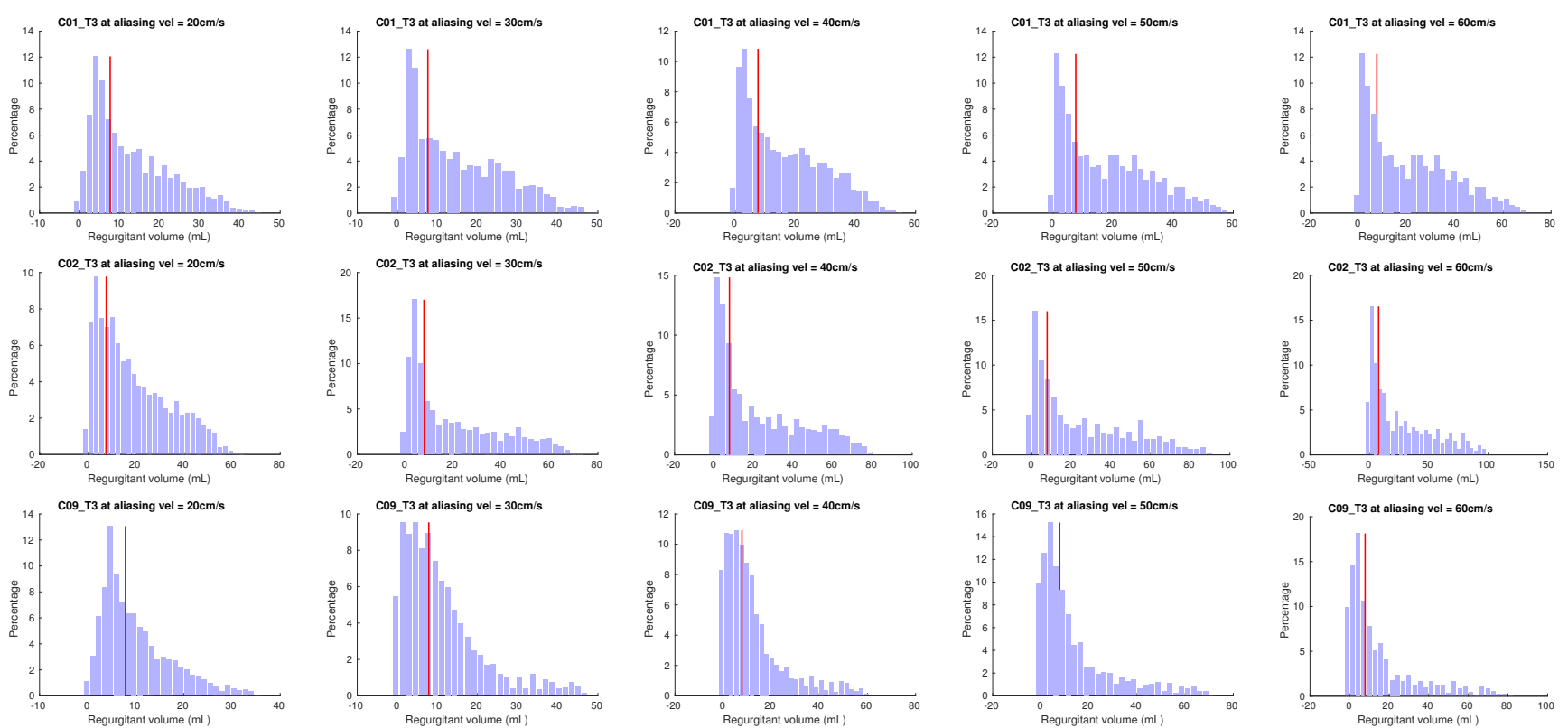

Figure C.18: PISA Estimates histogram for Type 3 cases 